Scientific Visualization, 2020, volume 12, number 2, pages 21 - 36, DOI: 10.26583/sv.12.2.03

\title{
Algebraic methods for coloring cubic graphs
}

\author{
S.V. Kurapovi,A, M.V. Davidovsky²,B, A.V. Tolok33,C \\ A Zaporozhye National University, Ukraine \\ в Zaporozhye Institute of Postgraduate Pedagogical Education, Ukraine \\ C Moscow State Technological University «STANKIN», Russia \\ ${ }^{1}$ ORCID: oooo-0003-4563-7227, lilili5050@rambler.ru \\ 2 ORCID: 00oo-0002-9472-3351, m.davidovsky@gmail.com \\ 3 ORCID: 0000-0002-7257-9029, a.tolok@stankin.ru
}

\begin{abstract}
The article addresses algebraic methods for coloring arbitrary cubic graphs. The results are partially based on the corollaries of the Tait theorem. In the article, the authors propose using a fourth-order Klein group transform in order to formally describe the coloring of a cubic graph. The transition to graph coloring is done by coloring the edges of basis cycles. Overall, the mathematical framework for describing topological graph drawing is presented and formally described in the article. Based on the edge coloring, the formation of colored disks and the mathematical description of the operation of colored disks rotation with subsequent recoloring of the edges are considered. It is shown that the operation of rotating color disks can be represented as a ring sum (addition modulo 2) of cycles. In order to unambiguously describe the representation of colored disks by means of basis cycles, the authors introduce the concept of embeddability of colored disks. For clarity, the authors provide several examples illustrating the application of colored disks rotation operation to concrete cubic graphs. The relation between the system of induced cycles generated by the rotation of graph vertices and the coloring of 2-factors of the cubic graph is established in the present study. It is shown that the ring sum of all cycles included in the colored 2-factors of the graph is an empty set. The article also addresses the issues of coloring non-planar cubic graphs. The relationship between basis cycles and a rim in a non-planar cubic graph and a ring sum of colored 2-factors is explicitly shown in the article. In addition, the relationship between the colored vertex rotation of a plane cubic graph and the closed Heawood paths is revealed and formally described.
\end{abstract}

Keywords: graph, topological graph drawing, geometric image of graph drawing, rotation of graph vertices, isometric cycles, planarity.

\section{Introduction}

In [3], a theorem was proved on the existence of a colored disk passing through linked edges in a basis simple cycle of a planar correctly colored cubic graph $\mathbf{H}$. Based on the proved theorem, it was shown that the four-color problem can be represented as its consequence. The introduction of a new operation - the rotation of a colored disk - made it possible to recolor the edges in a plane colored cubic graph and construct a visual coloring algorithm. The presented method allows recursively color the edges of the subsequent plane cubic graph $\mathbf{H}$ with $n$ vertices in three colors based on the previous colored cubic graph with $n-2$ vertices.

As for coloring there is a relation between the maximal planar graph $\mathbf{G}^{\prime}$ and the planar cubic graph $\mathbf{H}$ dual to $\mathbf{G}^{\prime}$. This relation is set by the following theorem.

Theorem 1. (Tait) [7]. A bridge-free trivalent plane graph can be face-colored with 4 colors if and only if it can be edge-colored with 3 colors. 
In other words, let $\mathbf{H}$ be a plane homogeneous cubic graph without isthmuses; a necessary and sufficient condition for the possibility of such coloring the faces of the graph in four colors, in which no two adjacent faces are colored the same color, is that the chromatic class of graph $\mathbf{H}$ be equal to three [4].

Thus, the coloring of edges and faces of a plane cubic graph is adequate to the coloring of vertices and edges in a maximally plane graph $\mathbf{G}^{\prime}$. Therefore, it is convenient to consider only the process of coloring a plane graph.

Tait's theorem not only establishes a relation between the coloring of vertices of a maximally plane graph and the coloring of edges of a cubic graph dual to it, but also indicates the use of the fourth-order transformation of the Klein group for coloring a graph [2]. This transformation allows connecting in a single whole the coloring of faces of a plane cubic graph $\mathbf{H}$ in four colors and coloring of edges in three colors.

\begin{tabular}{l|llll}
+ & $\mathrm{O}$ & $\mathbf{1}$ & $\mathbf{2}$ & 3 \\
\hline $\mathrm{W}$ & $\mathrm{W}$ & $\mathrm{R}$ & $\mathrm{B}$ & $\mathrm{G}$ \\
$\mathrm{R}$ & $\mathrm{R}$ & $\mathrm{W}$ & $\mathrm{G}$ & $\mathrm{B}$ \\
$\mathrm{B}$ & $\mathrm{B}$ & $\mathrm{G}$ & $\mathrm{W}$ & $\mathrm{R}$ \\
$\mathrm{G}$ & $\mathrm{G}$ & $\mathrm{B}$ & $\mathrm{R}$ & $\mathrm{W}$
\end{tabular}

Further development of the methods of coloring edges and faces in a plane cubic graph revealed the existence of a close relationship between the coloring and the topological drawing of a plane graph. In this article, we consider an algebraic framework for coloring cubic graphs.

\section{Mathematical framework for describing the topological drawing of a graph}

Graph $\mathbf{G}=(\mathbf{V}, \mathbf{E})$ is generally defined by a set of vertices $\mathbf{V}$, a set of edges $\mathbf{E}$ and a triple predicate $\mathbf{P}$ in the form of adjacency (incidence) matrix [1,6] without describing a drawing of the graph. For a topological description of planar graph drawing we use the concept of graph vertex rotation introduced by G. Ringel [5].

Definition 1. Given a graph $\mathbf{G}$, a rotation of a vertex $A$ is an oriented cyclic order (or a cyclic permutation) of all arcs incident with $A$.

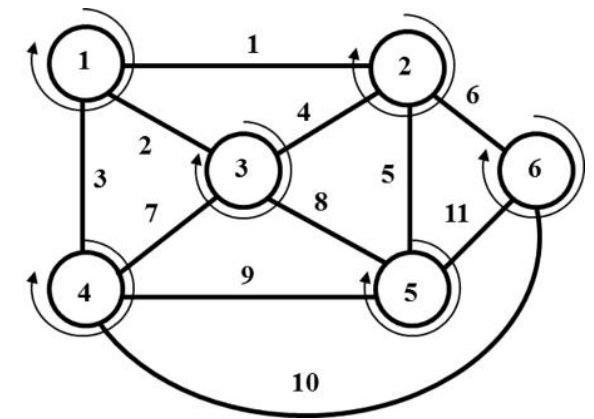

Fig. 1. Graph $\mathbf{G}$ and the rotation of its vertices.

Definition 2. A rotation of graph vertices $\sigma$ is the totality of the rotations of all vertices of G.

We will further represent the rotation of all vertices of a graph in the form of diagrams and denote it as $\sigma$. For example, for the graph drawing depicted in Fig. 1, the vertex rotation diagram has the following form:

$\begin{array}{lllll}\text { 1: } & 2 & 3 & 4 & \\ \text { 2: } & 6 & 5 & 3 & 1 \\ \text { 3: } & 2 & 5 & 4 & 1 \\ \text { 4: } & 1 & 3 & 5 & 6\end{array}$




$\begin{array}{lllll}\text { 5: } & 3 & 2 & 6 & 4 \\ \text { 6: } & 2 & 4 & 5 & \end{array}$

For convenience, the numbers in the vertex rotation diagram, indicate the numbers of vertices. In turn, the rotation of graph vertices induces a system of oriented simple cycles. In order to obtain such a system, we carry out a traversal along the edges, considering the transition from edge to edge according to the rotation of the vertices. We should note that during rotation each oriented edge always appears twice, the second time - in the opposite direction and in different cycle [5].

For example, for a plane graph $\mathbf{G}$ with rotation shown in Fig. 1, we have the following system of induced cycles: $\left\langle\mathrm{v}_{1}, \mathrm{v}_{3}, \mathrm{v}_{2}\right\rangle,\left\langle\mathrm{v}_{1}, \mathrm{v}_{4}, \mathrm{v}_{3}\right\rangle,\left\langle\mathrm{v}_{2}, \mathrm{v}_{3}, \mathrm{v}_{5}\right\rangle,\left\langle\mathrm{v}_{5}, \mathrm{v}_{3}, \mathrm{v}_{4}\right\rangle,\left\langle\mathrm{v}_{2}, \mathrm{v}_{5}, \mathrm{v}_{6}\right\rangle,\left\langle\mathrm{v}_{6}, \mathrm{v}_{5}, \mathrm{v}_{4}\right\rangle$, $\left\langle\mathrm{V}_{2}, \mathrm{~V}_{6}, \mathrm{v}_{4}, \mathrm{v}_{1}\right\rangle$. We will represent induced cycles in the form of a cyclic tuple of vertices describing a closed oriented path (either clockwise or counterclockwise, but always specifying this explicitly). Induced cycles can also be represented as sets of edges: $\left\{\mathrm{e}_{1}, \mathrm{e}_{2}, \mathrm{e}_{4}\right\},\left\{\mathrm{e}_{2}, \mathrm{e}_{3}, \mathrm{e}_{7}\right\}$, $\left\{\mathrm{e}_{7}, \mathrm{e}_{8}, \mathrm{e}_{9}\right\},\left\{\mathrm{e}_{4}, \mathrm{e}_{5}, \mathrm{e}_{8}\right\},\left\{\mathrm{e}_{5}, \mathrm{e}_{6}, \mathrm{e}_{11}\right\},\left\{\mathrm{e}_{9}, \mathrm{e}_{10}, \mathrm{e}_{11}\right\}$. Another induced cycle $\left\{\mathrm{e}_{1}, \mathrm{e}_{3}, \mathrm{e}_{6}, \mathrm{e}_{10}\right\}$ is the rim of the graph. It is equal to the ring sum of the cycles representing the boundaries of the graph drawing faces [5]. Thus, the rotation of graph vertices does not only represent a diagram of the vertices rotation, but it is also a system of simple cycles that are the boundaries of the faces (2-cells) of the plane drawing of the graph. On the other hand, such cycles form the basis of the subspace of cycles. Henceforth, we shall denote them as the basis cycles.

Induced oriented cycles can also be written as a closed sequence of oriented edges (arcs), e.g.: $<\mathrm{v} 1, \mathrm{v} 3, \mathrm{v} 2\rangle=(\mathrm{v} 1, \mathrm{v} 3)+(\mathrm{v} 3, \mathrm{v} 2)+(\mathrm{v} 2, \mathrm{v} 1)$.

Definition 3. A topological drawing of a plane graph is a graph with a given rotation of vertices.

\section{Construction of a colored disk}

The rotation of vertices $\sigma$ induces simple cycles that form a basis in the subspace of graph cycles $\mathbf{C}$. To establish the coloring of a plane cubic graph $\mathbf{H}$, it is necessary to color its edges (Fig. 2).

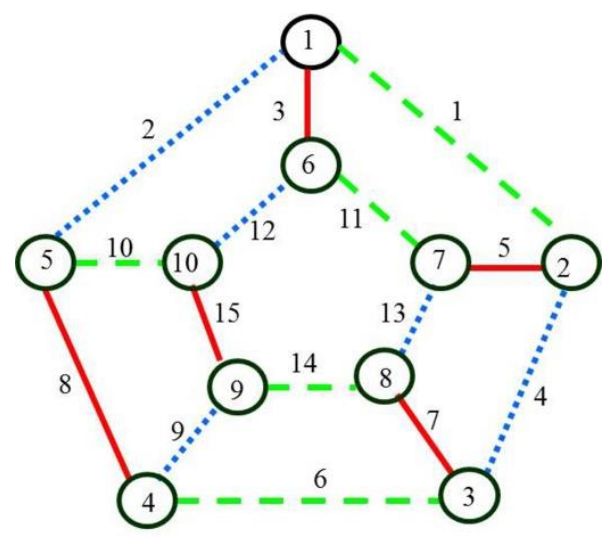

Fig. 2. Edge coloring in the plane cubic graph $\mathbf{H}_{1}$.

If the cubic graph $\mathbf{H}$ has a chromatic class of three, then its edges must be colored in three colors. The coloring assumes the presence of three edges of different colors for each vertex of graph $\mathbf{H}$. We call a cubic graph with chromatic class equal to three a colored plane cubic graph H. E.g., the cubic graph shown in Fig. 2 is essentially a colored cubic graph $\mathbf{H}$. Here, white color is designated with letter $\mathrm{W}$ or number $\mathrm{o}$, and on the graph drawings the edges of this color are represented with a dash-and-dot line. Red color is designated with letter R, or number 1, and on the graph drawings the edges of this color are represented with a solid line. Blue color is designated with letter B and number 2, and the edges of this color are represented with a dotted line on the graph drawings. In turn, green color is designated with letter G, or number 3 , and edges of this color are represented with dashed line. According to Tait's 
theorem, coloring of edges induces coloring of faces of a plane cubic graph $\mathbf{H}$, and coloring of faces, in turn, induces coloring of edges.

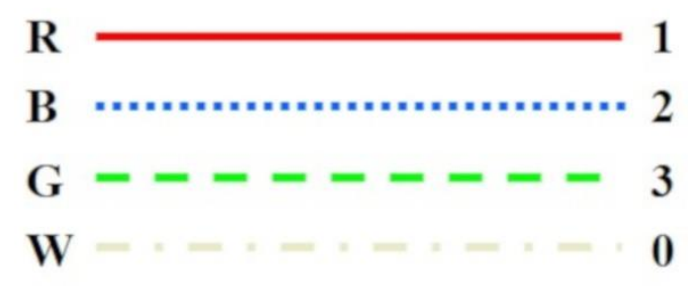

Fig. 3. Designation of colored lines.

Definition 4. In a colored plane cubic graph, a colored disk is a closed path of even length with edges of only two colors.

Thus, a red colored disk consists only of blue and green edges. A green colored disk consists only of blue and red edges. A blue colored disk consists only of green and red edges. The process of constructing a color disk can be seen as a sequence of connecting colored edges of two colors:

$$
\mathrm{D}=\langle\mathrm{v} 1, \mathrm{e} 1, \mathrm{v} 2, \mathrm{e} 2, \ldots, \mathrm{vp}-1, \mathrm{ep}-1 \mathrm{vp}, \mathrm{ep}, \mathrm{v} 1\rangle
$$

where $\mathrm{p}$ - is the length of a colored disk.

Let us denote a colored disk with the Latin letter D and the name of a color. For example, the red colored disk DR of the plane cubic graph $\mathbf{H}_{1}$ in Fig. 2 can be represented as a tuple:

$\mathrm{DR}=\langle\mathrm{v} 1, \mathrm{e} 2, \mathrm{v} 5, \mathrm{e} 10, \mathrm{v} 10, \mathrm{e} 12, \mathrm{v} 6, \mathrm{e} 11, \mathrm{v} 7, \mathrm{e} 13, \mathrm{v} 8, \mathrm{e} 14, \mathrm{v} 9, \mathrm{e} 9, \mathrm{v} 4, \mathrm{e} 6, \mathrm{v} 3, \mathrm{e} 4, \mathrm{v} 2, \mathrm{e} 1, \mathrm{v} 1>$; or taking into account the color of the edge indicated in square brackets:

$\mathrm{DR}=<\mathrm{e} 2[2], \mathrm{e} 10[3], \mathrm{e} 12[2], \mathrm{e} 11[3], \mathrm{e} 13[2], \mathrm{e} 14[3], \mathrm{eg}[2], \mathrm{e} 6[3], \mathrm{e} 4[2], \mathrm{e} 1[3]>$.

A colored disk in a plane cubic graph $\mathbf{H}$ can be represented as a ring addition of cycles [6]. Moreover, ring addition is carried out in the adjacency order of two cycles having a common edge of the disk's color. For example, the colored induced cycles of the graph $\mathbf{H}_{1}$ (which is shown in Fig. 2)

$$
\begin{array}{lll}
\mathrm{c} 1=\{\mathrm{e} 2[2], \mathrm{e} 3[1], \mathrm{e} 1 \mathrm{O}[3], \mathrm{e} 12[2]\} ; & \mathrm{c} 2=\{\mathrm{e} 1[3], \mathrm{e} 3[1], \mathrm{e} 5[1], \mathrm{e} 11[3]\} ; \\
\mathrm{c} 3=\{\mathrm{e} 4[2], \mathrm{e} 5[1], \mathrm{e} 7[1], \mathrm{e} 13[2]\} ; & \mathrm{c} 4=\{\mathrm{e} 6[3], \mathrm{e} 7[1], \mathrm{eg}[2], \mathrm{e} 14[3]\} ; \\
\mathrm{c} 5=\{\mathrm{e} 8[1], \mathrm{e} 9[2], \mathrm{e} 10[3], \mathrm{e} 15[1]\} ; & \mathrm{c} 6=\{\mathrm{e} 11[3], \mathrm{e} 12[2], \mathrm{e} 13[2], \mathrm{e} 14[3], \mathrm{e} 15[1]\} ; \\
\mathrm{co}=\{\mathrm{e} 1[3], \mathrm{e} 2[2], \mathrm{e} 4[2], \mathrm{e} 6[3], \mathrm{e} 8[1]\} . & &
\end{array}
$$

form the following colored 2-factors:

$$
\begin{aligned}
& \mathrm{Rc}=\mathrm{c} 1 \oplus \mathrm{c} 2 \oplus \mathrm{c} 3 \oplus \mathrm{c} 4=\{\mathrm{e} 2[2], \mathrm{e} 3[1], \mathrm{e} 10[3], \mathrm{e} 12[2]\} \oplus\{\mathrm{e} 1[3], \mathrm{e} 3[1], \mathrm{e} 5[1], \mathrm{e} 11[3]\} \oplus \\
& \oplus\{\mathrm{e} 4[2], \mathrm{e} 5[1], \mathrm{e} 7[1], \mathrm{e} 13[2]\} \oplus\{\mathrm{e} 6[3], \mathrm{e} 7[1], \mathrm{eg}[2], \mathrm{e} 14[3]\}= \\
& =\{\mathrm{e} 2[2], \mathrm{e} 10[3], \mathrm{e} 12[2], \mathrm{e} 1[3], \mathrm{e} 5[1], \mathrm{e} 11[3]\} \oplus\{\mathrm{e} 4[2], \mathrm{e} 5[1], \mathrm{e} 7[1], \mathrm{e} 13[2]\} \oplus \\
& \oplus\{\mathrm{e} 6[3], \mathrm{e} 7[1], \mathrm{eg}[2], \mathrm{e} 14[3]\}= \\
& =\{\mathrm{e} 2[2], \mathrm{e} 10[3], \mathrm{e} 12[2], \mathrm{e} 1[3], \mathrm{e} 11[3], \mathrm{e} 4[2], \mathrm{e} 7[1], \mathrm{e} 13[2]\} \oplus\{\mathrm{e} 6[3], \mathrm{e} 7[1], \mathrm{e} 9[2], \mathrm{e} 14[3]\}= \\
& =\{\mathrm{e} 2[2], \mathrm{e} 10[3], \mathrm{e} 12[2], \mathrm{e} 1[3], \mathrm{e} 11[3], \mathrm{e} 4[2], \mathrm{e} 13[2], \mathrm{e} 6[3], \mathrm{e} 9[2], \mathrm{e} 14[3]\}= \\
& =<\mathrm{e} 2[2], \mathrm{e} 1 \mathrm{O}[3], \mathrm{e} 12[2], \mathrm{e} 11[3], \mathrm{e} 13[2], \mathrm{e} 14[3], \mathrm{e} 9[2], \mathrm{e} 6[3], \mathrm{e} 4[2], \mathrm{e} 1[3]>; \\
& \mathrm{Bc}=\left(\mathrm{c}_{4} \oplus \mathrm{c} 5\right) \oplus(\mathrm{c} 2)=(\{\mathrm{e} 6[3], \mathrm{e} 7[1], \mathrm{eg}[2], \mathrm{e} 14[3]\} \oplus\{\mathrm{e} 8[1], \mathrm{e} 9[2], \mathrm{e} 1 \mathrm{o}[3], \mathrm{e} 15[1]\}) \oplus \\
& \oplus(\{\mathrm{e} 1[3], \mathrm{e} 3[1], \mathrm{e} 5[1], \mathrm{e} 11[3]\})=(\{\mathrm{e} 6[3], \mathrm{e} 7[1], \mathrm{e} 14[3], \mathrm{e} 8[1], \mathrm{e} 1 \mathrm{o}[3], \mathrm{e} 15[1]\}) \oplus \\
& \oplus(\{\mathrm{e} 1[3], \mathrm{e} 3[1], \mathrm{e} 5[1], \mathrm{e} 11[3]\})=(<\mathrm{e} 6[3], \mathrm{e} 7[1], \mathrm{e} 14[3], \mathrm{e} 15[1], \mathrm{e} 1 \mathrm{o}[3], \mathrm{e} 8[1]>), \\
& \text { (<e1[3],e5[1],e11[3],e3[1]>); } \\
& \mathrm{Gc}=(\mathrm{c} 1 \oplus \mathrm{c} 5) \oplus(\mathrm{c} 3)=(\{\mathrm{e} 2[2], \mathrm{e} 3[1], \mathrm{e} 10[3], \mathrm{e} 12[2]\} \oplus\{\mathrm{e} 8[1], \mathrm{e} 9[2], \mathrm{e} 10[3], \mathrm{e} 15[1]\}) \oplus \\
& \oplus(\{\mathrm{e} 4[2], \mathrm{e} 5[1], \mathrm{e} 7[1], \mathrm{e} 13[2]\})=(\{\mathrm{e} 2[2], \mathrm{e} 3[1], \mathrm{e} 12[2], \mathrm{e} 8[1], \mathrm{eg}[2], \mathrm{e} 15[1]\}) \oplus \\
& \oplus(\{\mathrm{e} 4[2], \mathrm{e} 5[1], \mathrm{e} 7[1], \mathrm{e} 13[2]\})=(<\mathrm{e} 2[2], \mathrm{e} 3[1], \mathrm{e} 12[2], \mathrm{e} 15[1], \mathrm{e} 9[2], \mathrm{e} 8[1]>), \\
& \text { (<e13[2],e5[1],e4[2],e } 7[1]>\text { ). }
\end{aligned}
$$


Since all edges are involved in the coloring of a plane graph twice (according to MacLane's theorem), then it follows from the construction:

Corollary 1. The ring sum (addition modulo 2) of all the edges included in the colored disks is an empty set.

$$
\sum_{i=1}^{m} e_{i}=\varnothing
$$

A special case is represented by white cycles that are not included in expression (2), where the edges can be colored in three colors. For our example:

$\mathrm{W}_{\mathrm{c}}=\mathrm{c}_{6} \oplus \mathrm{c}_{\mathrm{o}}=\left(\left\{\mathrm{e}_{11}[3], \mathrm{e}_{12}[2], \mathrm{e}_{13}[2], \mathrm{e}_{14}[3], \mathrm{e}_{15}[1]\right\}\right) \oplus\left(\left\{\mathrm{e}_{1}[3], \mathrm{e}_{2}[2], \mathrm{e}_{4}[2], \mathrm{e}_{6}[3], \mathrm{e}_{8}[1]\right\}\right)$.

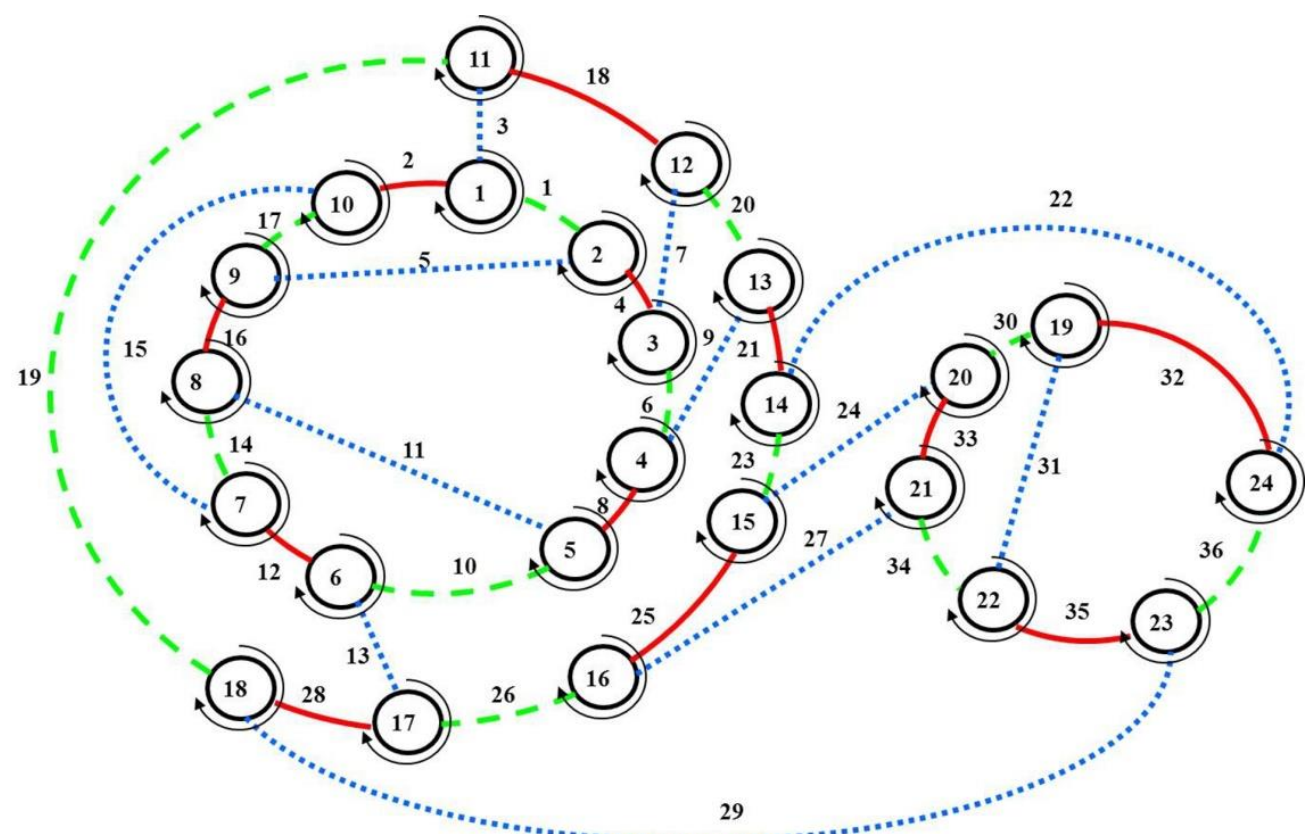

Fig. 4. The drawing of a colored plane cubic graph $\mathbf{H}_{2}$.

While the formation of color disks as a closed path of edges is carried out unambiguously, the formation of color disks by cycles has its own specific features. For illustration, let's consider the following drawing of a colored graph $\mathbf{H}_{2}$ (Fig. 4). For a given cubic graph $\mathbf{H}_{2}$, we select the following basis cycles:

$\mathrm{c} 1=\{\mathrm{e} 1[3], \mathrm{e} 2[1], \mathrm{e} 5[2], \mathrm{e} 17[3]\}, \mathrm{c} 2=\{\mathrm{e} 4[1], \mathrm{e} 5[2], \mathrm{e} 6[3], \mathrm{e} 8[1], \mathrm{e} 11[3], \mathrm{e} 16[1]\}$,

$\mathrm{c} 3=\{\mathrm{e} 10[3], \mathrm{e} 11[2], \mathrm{e} 12[1], \mathrm{e} 14[3]\}, \mathrm{c} 4=\{\mathrm{e} 14[3], \mathrm{e} 15[2], \mathrm{e} 16[1], \mathrm{e} 17[3]\}$,

$\mathrm{c} 5=\{\mathrm{e} 2[1], \mathrm{e} 3[2], \mathrm{e} 12[1], \mathrm{e} 13[2], \mathrm{e} 15[2], \mathrm{e} 19[3], \mathrm{e} 28[1]\}$,

$\mathrm{c} 6=\{\mathrm{e} 8[1], \mathrm{e} 9[2], \mathrm{e} 10[3], \mathrm{e} 13[2], \mathrm{e} 21[1], \mathrm{e} 23[3], \mathrm{e} 25[1], \mathrm{e} 26[3]\}, \mathrm{c} 7=\{\mathrm{e} 6[3], \mathrm{e} 7[2], \mathrm{e}$ [2], $\mathrm{e} 20[3]\}$,

$\mathrm{c} 8=\{\mathrm{e} 1[3], \mathrm{e} 3[2], \mathrm{e} 4[1], \mathrm{e} 7[2], \mathrm{e} 18[1]\}, \mathrm{c9}=\{\mathrm{e} 22[2], \mathrm{e} 23[3], \mathrm{e} 24[2], \mathrm{e} 3 \mathrm{O}[3], \mathrm{e} 32[1]\}$,

$\mathrm{c} 10=\{\mathrm{e} 24[2], \mathrm{e} 25[1], \mathrm{e} 27[2], \mathrm{e} 33[1]\}, \mathrm{c} 11=\{\mathrm{e} 26[3], \mathrm{e} 27[2], \mathrm{e} 28[1], \mathrm{e} 29[2], \mathrm{e} 34[3], \mathrm{e} 35[1]\}$,

$\mathrm{c} 12=\{\mathrm{e} 3 \mathrm{O}[3], \mathrm{e} 31[2], \mathrm{e} 33[1], \mathrm{e} 34[3]\}, \mathrm{c} 13=\{\mathrm{e} 31[2], \mathrm{e} 32[1], \mathrm{e} 35[1], \mathrm{e} 36[3]\}$.

Let's form the rim of the graph: $\mathrm{c}_{\mathrm{o}}=\left\{\mathrm{e}_{18}[1], \mathrm{e}_{19}[3], \mathrm{e}_{20}[3], \mathrm{e}_{21}[1], \mathrm{e}_{22}[2], \mathrm{e}_{29}[2], \mathrm{e}_{36}[3]\right\}$.

Next, we consider the case of the formation of a colored disk DB consisting of the following cycles:

$\mathrm{DB}=\mathrm{c}_{4} \oplus \mathrm{c}_{5} \oplus \mathrm{c}_{6} \oplus \mathrm{c}_{7} \oplus \mathrm{c}_{8}=\left\{\mathrm{e}_{14}[3], \mathrm{e}_{15}[2], \mathrm{e}_{16}[1], \mathrm{e}_{17}[3]\right\} \oplus$

$\oplus\left\{\mathrm{e}_{2}[1], \mathrm{e}_{3}[2], \mathrm{e}_{12}[1], \mathrm{e}_{13}[2], \mathrm{e}_{15}[2], \mathrm{e}_{19}[3], \mathrm{e}_{28}[1]\right\} \oplus$

$\oplus\left\{\mathrm{e}_{8}[1], \mathrm{e}_{9}[2], \mathrm{e}_{10}[3], \mathrm{e}_{13}[2], \mathrm{e}_{21}[1], \mathrm{e}_{23}[3], \mathrm{e}_{25}[1], \mathrm{e}_{26}[3]\right\} \oplus$

$\oplus\left\{\mathrm{e}_{6}[3], \mathrm{e}_{7}[2], \mathrm{e}_{9}[2], \mathrm{e}_{20}[3]\right\} \oplus\left\{\mathrm{e}_{1}[3], \mathrm{e}_{3}[2], \mathrm{e}_{4}[1], \mathrm{e}_{7}[2], \mathrm{e}_{18}[1]\right\}=$

$=\left\{\mathrm{e}_{14}, \mathrm{e}_{16}, \mathrm{e}_{17}, \mathrm{e}_{2}, \mathrm{e}_{12}, \mathrm{e}_{8}, \mathrm{e}_{10}, \mathrm{e}_{6}, \mathrm{e}_{1}, \mathrm{e}_{4}, \mathrm{e}_{19}, \mathrm{e}_{28}, \mathrm{e}_{21}, \mathrm{e}_{23}, \mathrm{e}_{25}, \mathrm{c}_{26}, \mathrm{e}_{20}, \mathrm{e}_{18}\right\}=$

$=<\mathrm{e}_{14}[3], \mathrm{e}_{16}[1], \mathrm{e}_{17}[3], \mathrm{e}_{2}[1], \mathrm{e}_{1}[3], \mathrm{e}_{4}[1], \mathrm{e}_{6}[3], \mathrm{e}_{8}[1], \mathrm{e}_{10}[3], \mathrm{e}_{12}[1]>\oplus$

$<\mathrm{e}_{19}[3], \mathrm{e}_{28}[1], \mathrm{e}_{26}[3], \mathrm{e}_{25}[1], \mathrm{e}_{23}[3], \mathrm{e}_{21}[1], \mathrm{e}_{20}[3], \mathrm{e}_{18}[1]>$. 
As one can see, this set of cycles forms two unicolored disks. For the unambiguous formation of a color disk, we need to add cycles $\mathrm{c}_{1}, \mathrm{c}_{2}, \mathrm{c}_{3}$, which in turn also unambiguously form a colored disk.

For the graph presented in Fig. 6, the colored disks before rotation are as follows:

$\mathrm{DB}_{1}=\mathrm{c}_{1} \oplus \mathrm{c}_{2} \oplus \mathrm{c}_{3}=<\mathrm{e}_{14}[3], \mathrm{e}_{16}[1], \mathrm{e}_{17}[3], \mathrm{e}_{2}[1], \mathrm{e}_{1}[3], \mathrm{e}_{4}[1], \mathrm{e}_{6}[3], \mathrm{e}_{8}[1], \mathrm{e}_{10}[3], \mathrm{e}_{12}[1]>;$

$\mathrm{DB}_{2}=\mathrm{c}_{4} \oplus \mathrm{c}_{5} \oplus \mathrm{c}_{6} \oplus \mathrm{c}_{7} \oplus \mathrm{c}_{8} \oplus \mathrm{c}_{1} \oplus \mathrm{c}_{2} \oplus \mathrm{c}_{3}=$

$=\left\langle\mathrm{e}_{19}[3], \mathrm{e}_{28}[1], \mathrm{e}_{26}[3], \mathrm{e}_{25}[1], \mathrm{e}_{23}[3], \mathrm{e}_{21}[1], \mathrm{e}_{20}[3], \mathrm{e}_{18}[1]>\right.$.

$\mathrm{DB}_{3}=\mathrm{c}_{12} \oplus \mathrm{c}_{13}=<\mathrm{e}_{30}[3], \mathrm{e}_{32}[1], \mathrm{e}_{36}[3], \mathrm{e}_{35}[1], \mathrm{e}_{34}[3], \mathrm{e}_{33}[1]>;$

Thus, the concept of embeddability of colored disks emerges. Considering the cycles of the graph $\mathbf{H}_{2}$, we can state that the cycles of the colored disk $\mathrm{DB}_{1}$ are embedded into the colored disk $\mathrm{DB}_{2}$.

Let's construct all the colored disks of the cubic graph $\mathrm{H}_{2}$ (Fig. 4).

$\mathrm{DR}_{1}=\mathrm{c}_{1} \oplus \mathrm{c}_{5} \oplus \mathrm{c}_{3} \oplus \mathrm{c}_{11} \oplus \mathrm{c}_{13} \oplus \mathrm{c}_{9}=$

$=<\mathrm{e}_{19}[3], \mathrm{e}_{29}[2], \mathrm{e}_{36}[3], \mathrm{e}_{22}[2], \mathrm{e}_{23}[3], \mathrm{e}_{24}[2], \mathrm{e}_{30}[3], \mathrm{e}_{31}[2], \mathrm{e}_{34}[3], \mathrm{e}_{27}[2], \mathrm{e}_{26}[3], \mathrm{e}_{13}[2], \mathrm{e}_{10}[3]$,

$\mathrm{e}_{11}[2], \mathrm{e}_{14}[3], \mathrm{e}_{15}[2], \mathrm{e}_{17}[3], \mathrm{e}_{5}[2], \mathrm{e}_{1}[3], \mathrm{e}_{3}[2]>;$

$\mathrm{DR}_{2}=\mathrm{c}_{7}=\left\langle\mathrm{e}_{7}[2], \mathrm{e}_{20}[3], \mathrm{e}_{9}[2], \mathrm{e}_{6}[3]>;\right.$

$\mathrm{DG}_{1}=\mathrm{c}_{10}=\left\langle\mathrm{e}_{24}[2], \mathrm{e}_{33}[1], \mathrm{e}_{27}[2], \mathrm{e}_{25}[1]>\right.$;

$\mathrm{DG}_{2}=\mathrm{c}_{1} \oplus \mathrm{c}_{3} \oplus \mathrm{c}_{4} \oplus \mathrm{c}_{6} \oplus \mathrm{c}_{8} \oplus \mathrm{c}_{9} \oplus \mathrm{c}_{10} \oplus \mathrm{c}_{11} \oplus \mathrm{c}_{12}=$

$=<\mathrm{e}_{18}[1], \mathrm{e}_{3}[2], \mathrm{e}_{2}[1], \mathrm{e}_{15}[2], \mathrm{e}_{12}[1], \mathrm{e}_{13}[2], \mathrm{e}_{28}[1], \mathrm{e}_{29}[2], \mathrm{e}_{35}[1], \mathrm{e}_{31}[2], \mathrm{e}_{32}[1], \mathrm{e}_{22}[2], \mathrm{e}_{21}[1]$, $\mathrm{e}_{9}[2], \mathrm{e}_{8}[1], \mathrm{e}_{11}[2], \mathrm{e}_{16}[1], \mathrm{e}_{5}[2], \mathrm{e}_{4}[1], \mathrm{e}_{7}[2]>;$

Notice that the disk $\mathrm{DG}_{2}$ is embeddable into the disk $\mathrm{DG}_{1}$.

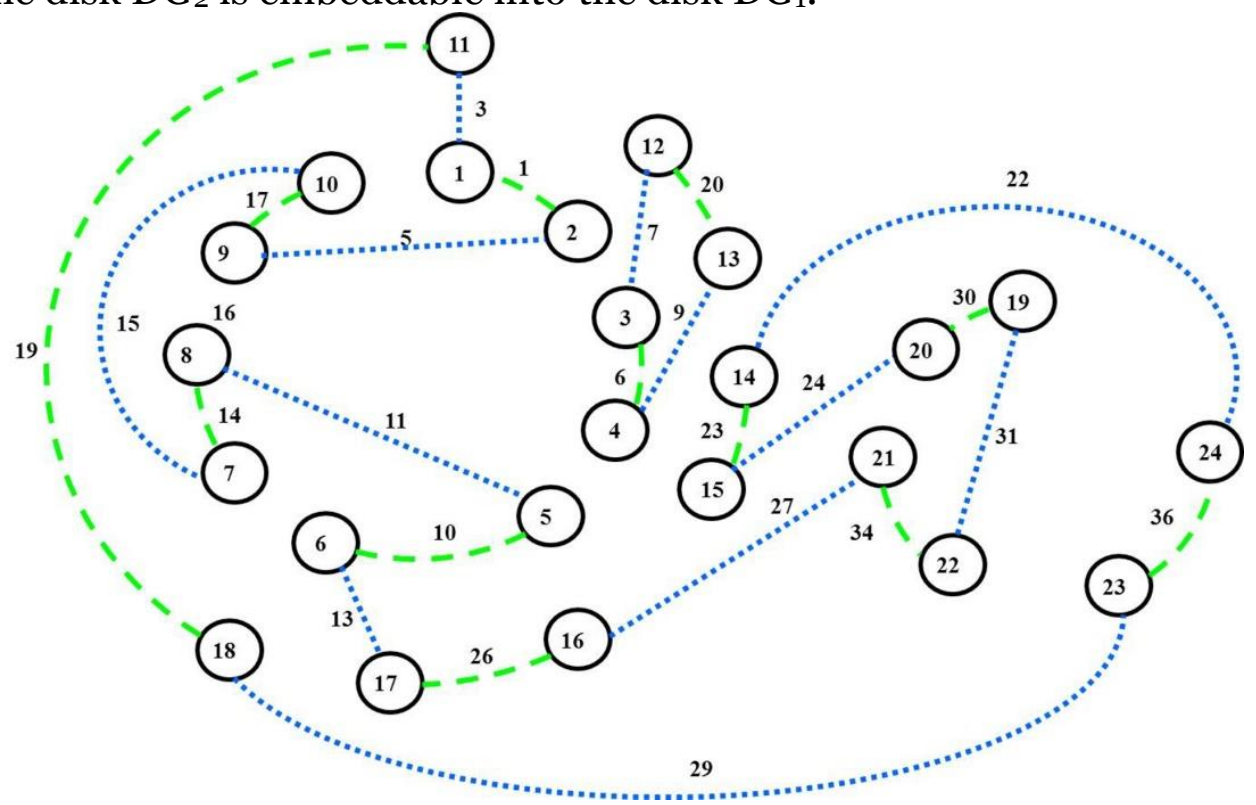

Fig. 5. Red 2-factor before rotation. 

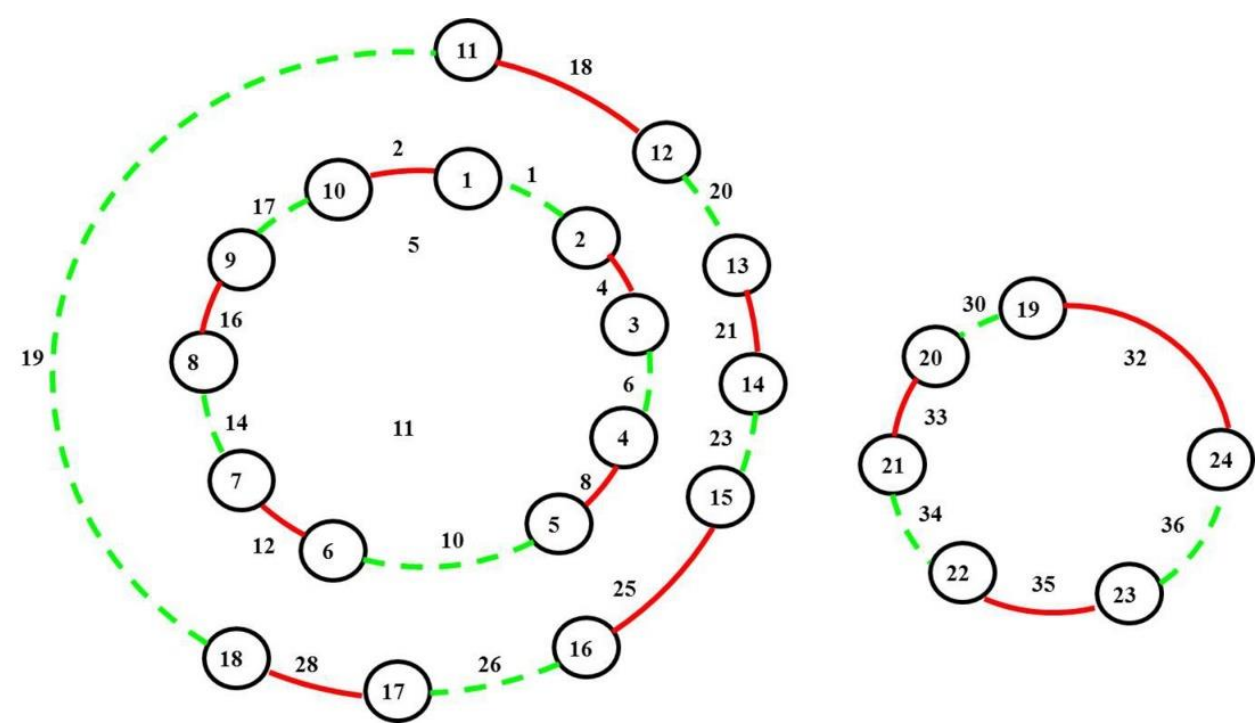

Fig. 6. Blue 2-factor before rotation.

$\mathrm{W}_{\mathrm{c}}=\mathrm{c}_{\mathrm{o}}=\left\langle\mathrm{e}_{18}[1], \mathrm{e}_{19}[3], \mathrm{e}_{2 \mathrm{o}}[3], \mathrm{e}_{21}[1], \mathrm{e}_{22}[2], \mathrm{e}_{29}[2], \mathrm{e}_{36}[3]>;\right.$

Colored 2-factors are formed as a ring sum of the corresponding colored disks. For instance, for the graph $\mathbf{H}_{2}$, the red disks are shown in Fig. 5 .

$\mathrm{R}_{\mathrm{c}}=\mathrm{DR}_{1} \oplus \mathrm{DR}_{2}=\left(\mathrm{c}_{1} \oplus \mathrm{c}_{5} \oplus \mathrm{c}_{3} \oplus \mathrm{c}_{11} \oplus \mathrm{c}_{13} \oplus \mathrm{c}_{9}\right) \oplus\left(\mathrm{c}_{7}\right)=$

$=<\mathrm{e}_{19}[3], \mathrm{e}_{29}[2], \mathrm{e}_{36}[3], \mathrm{e}_{22}$ [2], $\mathrm{e}_{23}[3], \mathrm{e}_{24}[2], \mathrm{e}_{30}[3], \mathrm{e}_{31}[2], \mathrm{e}_{34}[3], \mathrm{e}_{27}[2], \mathrm{e}_{26}[3], \mathrm{e}_{13}[2], \mathrm{e}_{10}[3], \mathrm{e}_{11}[2]$, $\mathrm{e}_{14}[3], \mathrm{e}_{15}[2], \mathrm{e}_{17}[3], \mathrm{e}_{5}[2], \mathrm{e}_{1}[3], \mathrm{e}_{3}[2]>\oplus<\mathrm{e}_{7}[2], \mathrm{e}_{20}[3], \mathrm{e}_{9}[2], \mathrm{e}_{6}[3]>$;

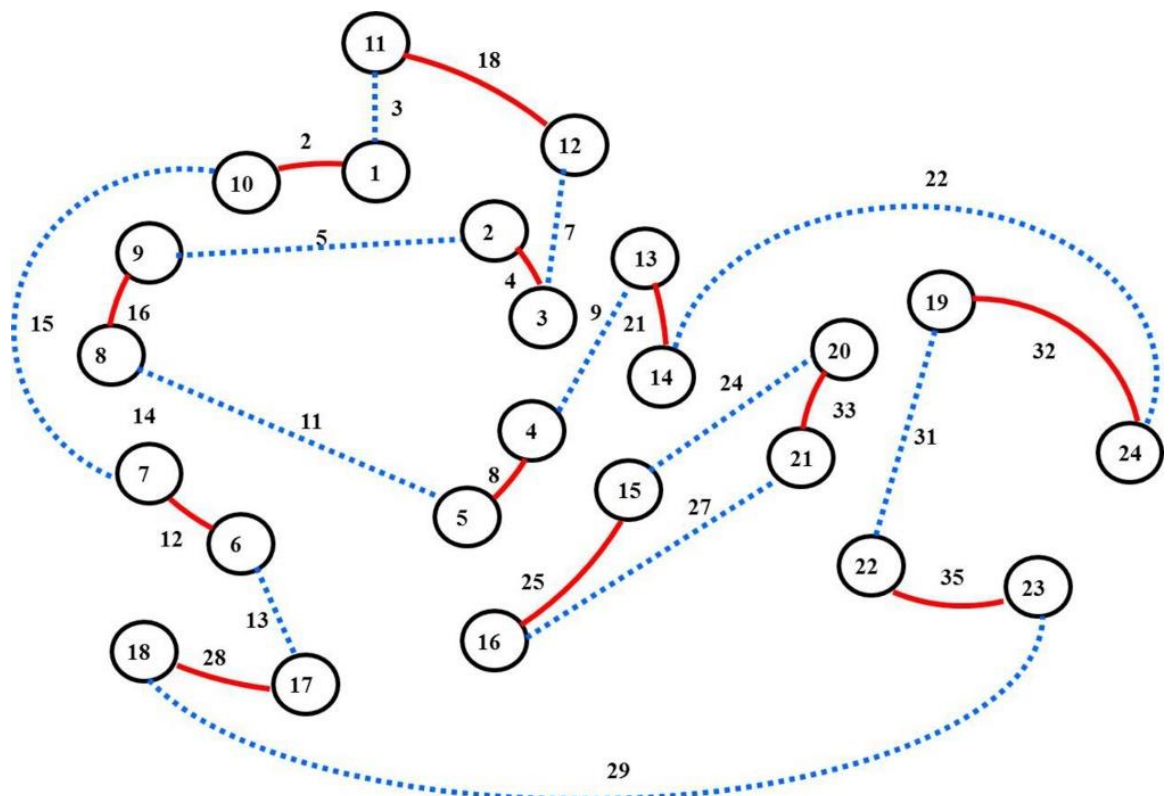

Fig. 7. Green 2-factor before rotation. 


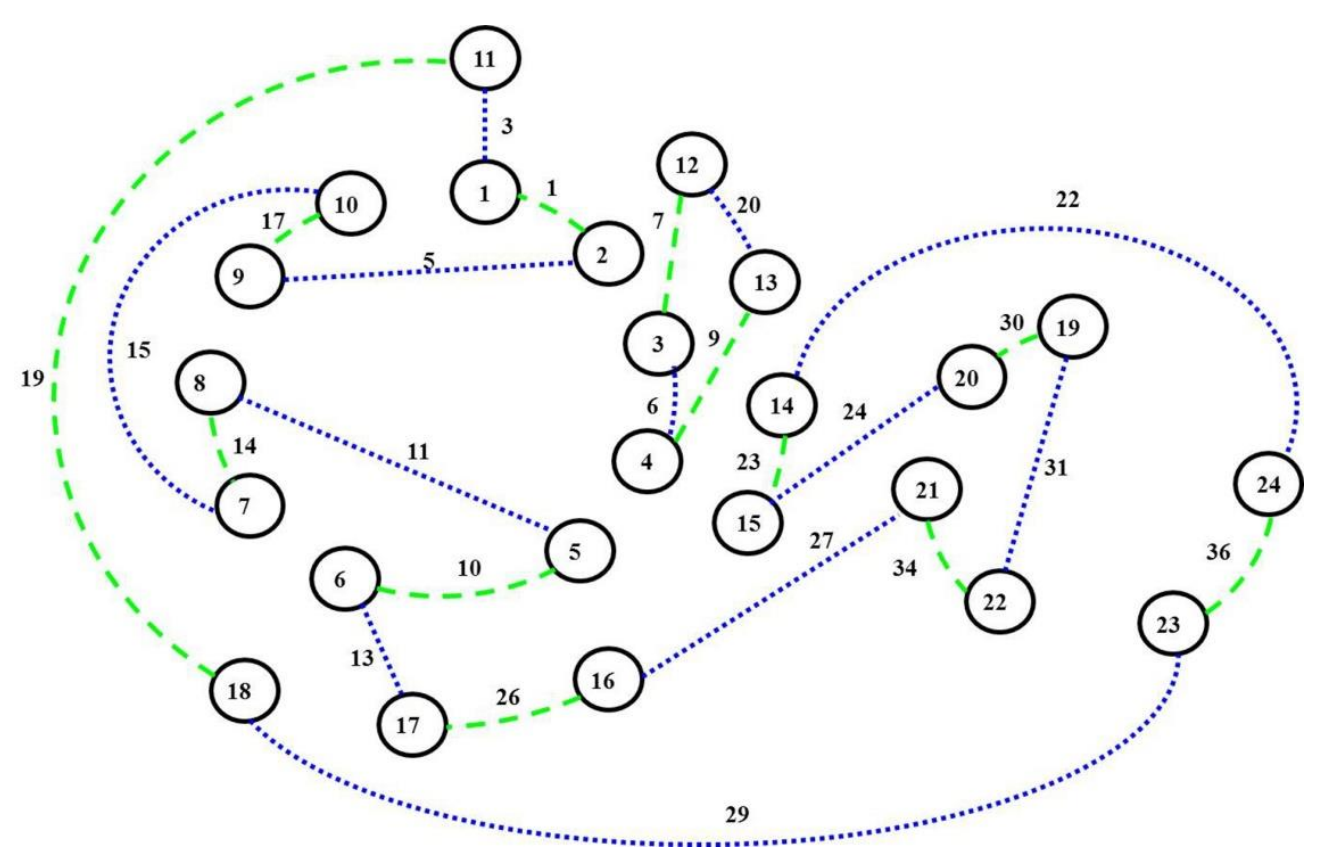

Fig. 8. Red 2-factor after rotation.

The blue disks are shown in Fig. 6 .

$\mathrm{B}_{\mathrm{c}}=\mathrm{DB}_{1} \oplus \mathrm{DB}_{2} \oplus \mathrm{DB}_{3}=\left(\mathrm{c}_{1} \oplus \mathrm{c}_{2} \oplus \mathrm{c}_{3}\right) \oplus\left(\mathrm{c}_{4} \oplus \mathrm{c}_{5} \oplus \mathrm{c}_{6} \oplus \mathrm{c}_{7} \oplus \mathrm{c}_{8} \oplus \mathrm{c}_{1} \oplus \mathrm{c}_{2} \oplus \mathrm{c}_{3}\right) \oplus$

$\oplus\left(\mathrm{c}_{12} \oplus \mathrm{c}_{13}\right)=\left(\mathrm{c}_{4} \oplus \mathrm{c}_{5} \oplus \mathrm{c}_{6} \oplus \mathrm{c}_{7} \oplus \mathrm{c}_{8}\right) \oplus\left(\mathrm{c}_{12} \oplus \mathrm{c}_{13}\right)=$

$=<\mathrm{e}_{14}[3], \mathrm{e}_{16}[1], \mathrm{e}_{17}[3], \mathrm{e}_{2}[1], \mathrm{e}_{1}[3], \mathrm{e}_{4}[1], \mathrm{e}_{6}[3], \mathrm{e}_{8}[1], \mathrm{e}_{10}[3], \mathrm{e}_{12}[1]>\oplus$

$\oplus<\mathrm{e}_{18}[1], \mathrm{e}_{20}[3], \mathrm{e}_{21}[1], \mathrm{e}_{23}[3], \mathrm{e}_{25}[1], \mathrm{e}_{26}[3], \mathrm{e}_{28}[1], \mathrm{e}_{19}[3]>\oplus$

$\oplus<\mathrm{e}_{30}[3], \mathrm{e}_{32}[1], \mathrm{e}_{36}[3], \mathrm{e}_{35}[1], \mathrm{e}_{34}[3], \mathrm{e}_{33}[1]>$;

The green disks are shown in Fig. 7.

$\mathrm{G}_{\mathrm{c}}=\mathrm{DG}_{1} \oplus \mathrm{DG}_{2}=\left(\mathrm{c}_{8} \oplus \mathrm{c}_{1} \oplus \mathrm{c}_{4} \oplus \mathrm{c}_{3} \oplus \mathrm{c}_{6} \oplus \mathrm{c}_{11} \oplus \mathrm{c}_{12} \oplus \mathrm{c}_{9} \oplus \mathrm{c}_{10}\right) \oplus\left(\mathrm{c}_{10}\right)=$

$=\mathrm{c}_{8} \oplus \mathrm{c}_{1} \oplus \mathrm{c}_{4} \oplus \mathrm{c}_{3} \oplus \mathrm{c}_{6} \oplus \mathrm{c}_{11} \oplus \mathrm{c}_{12} \oplus \mathrm{c}_{9}=$

$=<\mathrm{e}_{2}[1], \mathrm{e}_{15}[2], \mathrm{e}_{12}[1], \mathrm{e}_{13}[2], \mathrm{e}_{28}[1], \mathrm{e}_{29}[2], \mathrm{e}_{35}[1], \mathrm{e}_{31}[2], \mathrm{e}_{32}[1], \mathrm{e}_{22}[2], \mathrm{e}_{21}[1], \mathrm{e}_{9}[2], \mathrm{e}_{8}[1], \mathrm{e}_{11}[2]$,

$\mathrm{e}_{16}[1], \mathrm{e}_{5}[2], \mathrm{e}_{4}[1], \mathrm{e}_{7}[2], \mathrm{e}_{18}[1], \mathrm{e}_{3}[2]>\oplus<\mathrm{e}_{24}[2], \mathrm{e}_{33}[1], \mathrm{e}_{27}[2], \mathrm{e}_{25}[1]>$.

The ring sum of all colored disks of the same color forms a 2-factor of the same color. For example, in Fig. 5-7 the following colored 2-factors are shown:

$\mathbf{R}_{c}=\mathbf{D R}_{1} \oplus \mathbf{D R}_{2} ; \quad \mathbf{B}_{c}=\mathbf{D B}_{1} \oplus \mathbf{D B}_{2} \oplus \mathbf{D B}_{3} ; \quad \mathbf{G}_{c}=\mathbf{D G}_{1} \oplus \mathbf{D G}_{2}$

From the construction follows:

Corollary 2. The ring sum (adding modulo 2) of all cycles involved in the formation of colored 2-factors is an empty set:

$$
\mathrm{R}_{\mathrm{c}} \oplus \mathrm{B}_{\mathrm{c}} \oplus \mathrm{G}_{\mathrm{c}}=\varnothing
$$

The following expression establishes a relation between the colored 2-factors and the basis cycles of a plane cubic graph:

$$
2\left(\sum_{i=0}^{m-n+1} \mathbf{c}_{i} \oplus \mathbf{c}_{0}\right)=\left(\mathbf{R}_{c} \oplus \mathbf{B}_{c} \oplus \mathbf{G}_{c}\right) \oplus 2 \mathbf{W}_{c}
$$

Corollary 3. The doubled sum of the basis cycles of the topological drawing and the rim is equal to the ring sum of the cycles of the colored 2-factors plus the doubled sum of the white cycles.

\section{Rotation of colored disks}

By rotation of a colored Hamiltonian disk, we mean a change in the sequence of coloring of the edges of a given disk. Disk rotation changes other colored disks. 
Consider the rotation of the colored disk $\mathrm{DR}_{2}$. During rotation, only the colors of the edges $\mathrm{e}_{6}, \mathrm{e}_{7}, \mathrm{e}_{9}, \mathrm{e}_{20}$ belonging to the colored disk $\mathrm{DR}_{2}$ change. The colors of other edges do not change. To define new sequences in colored disks, we attach the cycles belonging to the disk $\mathrm{DR}_{2}$ to other colored 2-factors.

$\mathrm{R}_{\mathrm{c}}=\mathrm{DR}_{1} \oplus \mathrm{DR}_{2}=\left(\mathrm{c}_{1} \oplus \mathrm{c}_{5} \oplus \mathrm{c}_{3} \oplus \mathrm{c}_{11} \oplus \mathrm{c}_{13} \oplus \mathrm{c}_{9}\right) \oplus\left(\mathrm{c}_{7}\right)$;

$\mathrm{B}_{\mathrm{c}}=\mathrm{DB}_{1} \oplus \mathrm{DB}_{2} \oplus \mathrm{DB}_{3}=\left(\mathrm{c}_{4} \oplus \mathrm{c}_{5} \oplus \mathrm{c}_{6} \oplus \mathrm{c}_{7} \oplus \mathrm{c}_{8}\right) \oplus\left(\mathrm{c}_{12} \oplus \mathrm{c}_{13}\right) \oplus\left(\mathrm{c}_{7}\right) ;$

$\mathrm{G}_{\mathrm{c}}=\mathrm{DG}_{1} \oplus \mathrm{DG}_{2}=\left(\mathrm{c}_{8} \oplus \mathrm{c}_{1} \oplus \mathrm{c}_{4} \oplus \mathrm{c}_{3} \oplus \mathrm{c}_{6} \oplus \mathrm{c}_{11} \oplus \mathrm{c}_{12} \oplus \mathrm{c}_{9}\right) \oplus\left(\mathrm{c}_{7}\right)$;

As a result, we obtain the coloring of red colored disks (Fig. 8):

$\mathrm{R}_{\mathrm{c}}=\left(\mathrm{c}_{1} \oplus \mathrm{c}_{5} \oplus \mathrm{c}_{3} \oplus \mathrm{c}_{11} \oplus \mathrm{c}_{13} \oplus \mathrm{c}_{9}\right) \oplus\left(\mathrm{c}_{7}\right)=$

$=<\mathrm{e}_{19}[3], \mathrm{e}_{29}[2], \mathrm{e}_{36}[3], \mathrm{e}_{22}[2], \mathrm{e}_{23}[3], \mathrm{e}_{24}[2], \mathrm{e}_{30}[3], \mathrm{e}_{31}[2], \mathrm{e}_{34}[3], \mathrm{e}_{27}[2], \mathrm{e}_{26}[3], \mathrm{e}_{13}[2], \mathrm{e}_{10}[3], \mathrm{e}_{11}[2]$,

$\mathrm{e}_{14}[3], \mathrm{e}_{15}[2], \mathrm{e}_{17}[3], \mathrm{e}_{5}[2], \mathrm{e}_{1}[3], \mathrm{e}_{3}[2]>\oplus<\mathrm{e}_{7}[3], \mathrm{e}_{20}[2], \mathrm{e}_{9}[3], \mathrm{e}_{6}[2]>$;

blue colored disks (Fig. 9):

$\mathrm{B}_{\mathrm{c}}=\left(\mathrm{c}_{4} \oplus \mathrm{c}_{5} \oplus \mathrm{c}_{6} \oplus \mathrm{c}_{7} \oplus \mathrm{c}_{8}\right) \oplus\left(\mathrm{c}_{12} \oplus \mathrm{c}_{13}\right) \oplus\left(\mathrm{c}_{7}\right)=$

$=\left\{\mathrm{e}_{14}[3], \mathrm{e}_{15}[2], \mathrm{e}_{16}[1], \mathrm{e}_{17}[3]\right\} \oplus\left\{\mathrm{e}_{2}[1], \mathrm{e}_{3}[2], \mathrm{e}_{12}[1], \mathrm{e}_{13}[2], \mathrm{e}_{15}[2], \mathrm{e}_{19}[3], \mathrm{e}_{28}[1]\right\} \oplus$

$\left\{\mathrm{e}_{8}[1], \mathrm{e}_{9}[3], \mathrm{e}_{10}[3], \mathrm{e}_{13}[2], \mathrm{e}_{21}[1], \mathrm{e}_{23}[3], \mathrm{e}_{25}[1], \mathrm{e}_{26}[3]\right\} \oplus\left\{\mathrm{e}_{6}[2], \mathrm{e}_{7}[3], \mathrm{e}_{9}[3], \mathrm{e}_{20}[2]\right\} \oplus$

$\oplus\left\{\mathrm{e}_{1}[3], \mathrm{e}_{3}[2], \mathrm{e}_{4}[1], \mathrm{e}_{7}[3], \mathrm{e}_{18}[1]\right\} \oplus\left\{\mathrm{e}_{30}[3], \mathrm{e}_{31}[2], \mathrm{e}_{33}[1], \mathrm{e}_{34}[3]\right\} \oplus$

$\oplus\left\{\mathrm{e}_{31}[2], \mathrm{e}_{32}[1], \mathrm{e}_{35}[1], \mathrm{e}_{36}[3]\right\} \oplus\left\{\mathrm{e}_{6}[2], \mathrm{e}_{7}[3], \mathrm{e}_{9}[3], \mathrm{e}_{20}[2]\right\}=$

$=\left\{\mathrm{e}_{14}[3], \mathrm{e}_{16}[1], \mathrm{e}_{17}[3], \mathrm{e}_{2}[1], \mathrm{e}_{12}[1], \mathrm{e}_{19}[3], \mathrm{e}_{28}[1], \mathrm{e}_{8}[1], \mathrm{e}_{10}[3], \mathrm{e}_{21}[1], \mathrm{e}_{23}[3], \mathrm{e}_{25}[1], \mathrm{e}_{26}[3], \mathrm{e}_{1}[3]\right.$,

$\left.\mathrm{e}_{4}[1], \mathrm{e}_{7}[3], \mathrm{e}_{18}[1], \mathrm{e}_{30}[3], \mathrm{e}_{33}[1], \mathrm{e}_{34}[3], \mathrm{e}_{32}[1], \mathrm{e}_{35}[1], \mathrm{e}_{36}[3]\right\}=$

$=<\mathrm{e}_{14}[3], \mathrm{e}_{16}[1], \mathrm{e}_{17}[3], \mathrm{e}_{2}[1], \mathrm{e}_{1}[3], \mathrm{e}_{4}[1], \mathrm{e}_{7}[3], \mathrm{e}_{18}[1], \mathrm{e}_{19}[3], \mathrm{e}_{28}[1], \mathrm{e}_{26}[3], \mathrm{e}_{25}[1], \mathrm{e}_{23}[3]$,

$\mathrm{e}_{21}[1], \mathrm{e}_{9}[3], \mathrm{e}_{8}[1], \mathrm{e}_{10}[3], \mathrm{e}_{12}[1]>\oplus<\mathrm{e}_{30}[3], \mathrm{e}_{32}[1], \mathrm{e}_{36}[3], \mathrm{e}_{35}[1], \mathrm{e}_{34}[3], \mathrm{e}_{33}[1]>;$

green colored disks (Fig. 10):

$\mathrm{G}_{\mathrm{c}}=\left(\mathrm{c}_{8} \oplus \mathrm{c}_{1} \oplus \mathrm{c}_{4} \oplus \mathrm{c}_{3} \oplus \mathrm{c}_{6} \oplus \mathrm{c}_{11} \oplus \mathrm{c}_{12} \oplus \mathrm{c}_{9}\right) \oplus\left(\mathrm{c}_{7}\right)=\left\{\mathrm{e}_{1}[3], \mathrm{e}_{3}[2], \mathrm{e}_{4}[1], \mathrm{e}_{7}[3], \mathrm{e}_{18}[1]\right\} \oplus$ $\left\{\mathrm{e}_{1}[3], \mathrm{e}_{2}[1], \mathrm{e}_{5}[2], \mathrm{e}_{17}[3]\right\} \oplus\left\{\mathrm{e}_{14}[3], \mathrm{e}_{15}[2], \mathrm{e}_{16}[1], \mathrm{e}_{17}[3]\right\} \oplus\left\{\mathrm{e}_{10}[3], \mathrm{e}_{11}[2], \mathrm{e}_{12}[1], \mathrm{e}_{14}[3]\right\} \oplus$

$\oplus\left\{\mathrm{e}_{8}[1], \mathrm{e}_{9}[3], \mathrm{e}_{10}[3], \mathrm{e}_{13}[2], \mathrm{e}_{21}[1], \mathrm{e}_{23}[3], \mathrm{e}_{25}[1], \mathrm{e}_{26}[3]\right\} \oplus$

$\oplus\left\{\mathrm{e}_{26}[3], \mathrm{e}_{27}[2], \mathrm{e}_{28}[1], \mathrm{e}_{29}[2], \mathrm{e}_{34}[3], \mathrm{e}_{35}[1]\right\} \oplus$

$\oplus\left\{\mathrm{e}_{30}[3], \mathrm{e}_{31}[2], \mathrm{e}_{33}[1], \mathrm{e}_{34}[3]\right\} \oplus\left\{\mathrm{e}_{22}[2], \mathrm{e}_{23}[3], \mathrm{e}_{24}[2], \mathrm{e}_{30}[3], \mathrm{e}_{32}[1]\right\} \oplus$

$\oplus\left\{\mathrm{e}_{6}[2], \mathrm{e}_{7}[3], \mathrm{e}_{9}[3], \mathrm{e}_{20}[2]\right\}=\left\{\mathrm{e}_{15}[2], \mathrm{e}_{16}[1], \mathrm{e}_{11}[2], \mathrm{e}_{12}[1], \mathrm{e}_{8}[1], \mathrm{e}_{13}[2], \mathrm{e}_{21}[1], \mathrm{e}_{25}[1], \mathrm{e}_{27}[2]\right.$, $\mathrm{e}_{28}[1], \mathrm{e}_{29}[2], \mathrm{e}_{35}[1], \mathrm{e}_{31}[2], \mathrm{e}_{33}[1], \mathrm{e}_{22}[2], \mathrm{e}_{24}[2], \mathrm{e}_{32}[1], \mathrm{e}_{6}[2], \mathrm{e}_{18}[1], \mathrm{e}_{3}[2], \mathrm{e}_{4}[1], \mathrm{e}_{2}[1], \mathrm{e}_{5}[2]$, $\left.\mathrm{e}_{20}[2]\right\}=\left\langle\mathrm{e}_{15}[2], \mathrm{e}_{12}[1], \mathrm{e}_{13}[2], \mathrm{e}_{28}[1], \mathrm{e}_{29}[2], \mathrm{e}_{35}[1], \mathrm{e}_{31}[2], \mathrm{e}_{32}[1], \mathrm{e}_{22}[2], \mathrm{e}_{21}[1], \mathrm{e}_{20}[2], \mathrm{e}_{18}[1]\right.$, $\left.\left.\mathrm{e}_{3}[2], \mathrm{e}_{2}[1]\right\rangle \oplus<\mathrm{e}_{5}[2], \mathrm{e}_{4}[1], \mathrm{e}_{6}[2], \mathrm{e}_{8}[1], \mathrm{e}_{11}[2], \mathrm{e}_{16}[1]\right\rangle \oplus\left\langle\mathrm{e}_{24}[2], \mathrm{e}_{25}[1], \mathrm{e}_{27}[2], \mathrm{e}_{33}[1]>\right.$.

Next, we form the rim of the graph, $\mathrm{c}_{0}=\left\{\mathrm{e}_{18}[1], \mathrm{e}_{19}[3], \mathrm{e}_{20}[3], \mathrm{e}_{21}[1], \mathrm{e}_{22}[2], \mathrm{e}_{29}[2], \mathrm{e}_{36}[3]\right\}$.
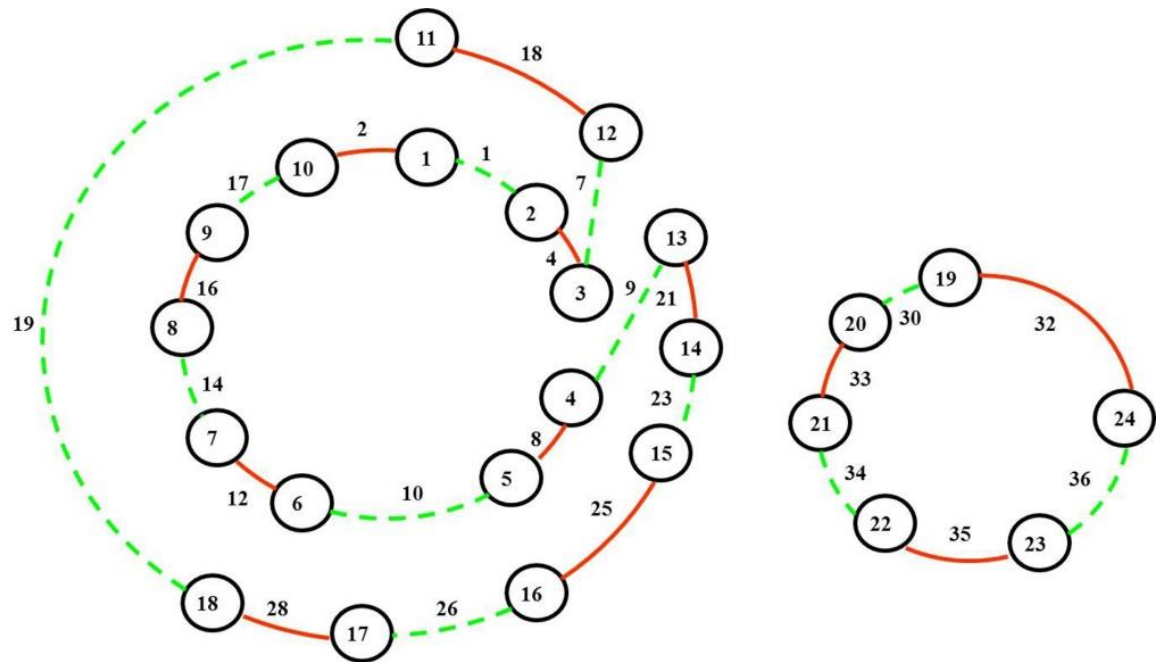

Fig. 9. Blue 2-factor after rotation. 


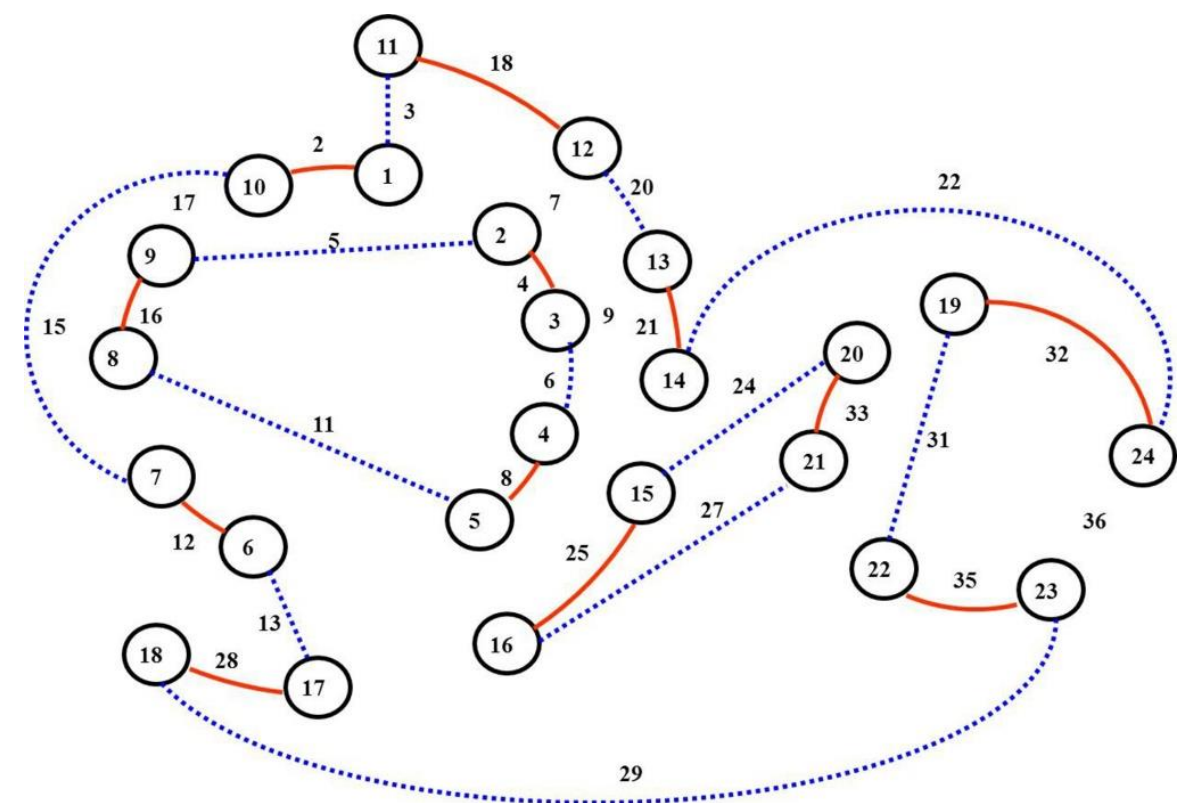

Fig. 10. Green 2-factor after rotation.

A rule for determining the composition of disks after rotation. To determine the composition of colored disks after rotating a colored disk $\mathrm{Y}$, it is necessary to recolor its edges and add cycles of colored disk $\mathrm{Y}$ to other colored 2-factors; then carry out the edge construction in accordance to (2).

Of interest are colored cubic graphs in which white colored disks have an even length and their total length is $2 \mathrm{~m} / 3$.

For the colored cubic graph $\mathbf{H}_{3}$ (Fig. 11), we select the following basis cycles:
$\mathrm{c}_{1}=\left\{\mathrm{e}_{2}[2], \mathrm{e}_{3}[1], \mathrm{e}_{8}[1], \mathrm{e}_{10}[2]\right\}$
$\mathrm{c}_{2}=\left\{\mathrm{e}_{1}[3], \mathrm{e}_{2}[2], \mathrm{e}_{4}[1], \mathrm{e}_{6}[3]\right\}$
$\mathrm{c}_{3}=\left\{\mathrm{e}_{9}[3], \mathrm{e}_{10}[2], \mathrm{e}_{11}[1], \mathrm{e}_{13}[3]\right\} ;$
$\mathrm{c}_{4}=\left\{\mathrm{e}_{4}[1], \mathrm{e}_{5}[2], \mathrm{e}_{7}[2], \mathrm{e}_{18}[1]\right\}$
$\mathrm{c}_{5}=\left\{\mathrm{e}_{11}[1], \mathrm{e}_{12}[2], \mathrm{e}_{14}[2], \mathrm{e}_{23}[1]\right\}$
$\mathrm{c}_{6}=\left\{\mathrm{e}_{6}[3], \mathrm{e}_{7}[2], \mathrm{e}_{8}[1], \mathrm{e}_{9}[3], \mathrm{e}_{12}[2], \mathrm{e}_{15}[3], \mathrm{e}_{17}[1], \mathrm{e}_{24}[3]\right\} ;$
$\mathrm{c}_{7}=\left\{\mathrm{e}_{16}[2], \mathrm{e}_{17}[1], \mathrm{e}_{20}[1], \mathrm{e}_{22}[2]\right\} ;$
$\mathrm{c}_{8}=\left\{\mathrm{e}_{15}[3], \mathrm{e}_{16}[2], \mathrm{e}_{18}[1], \mathrm{e}_{19}[3]\right\}$;
$\mathrm{c}_{9}=\left\{\mathrm{e}_{21}[3], \mathrm{e}_{22}[2], \mathrm{e}_{23}[1], \mathrm{e}_{24}[3]\right\}$.

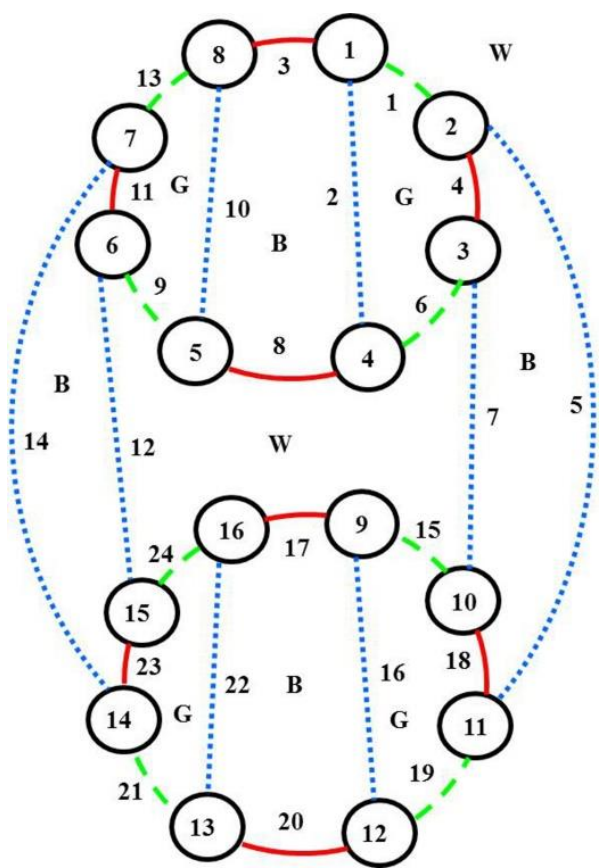

Fig. 11. Coloring of the cubic graph $\mathbf{H}_{3}$.

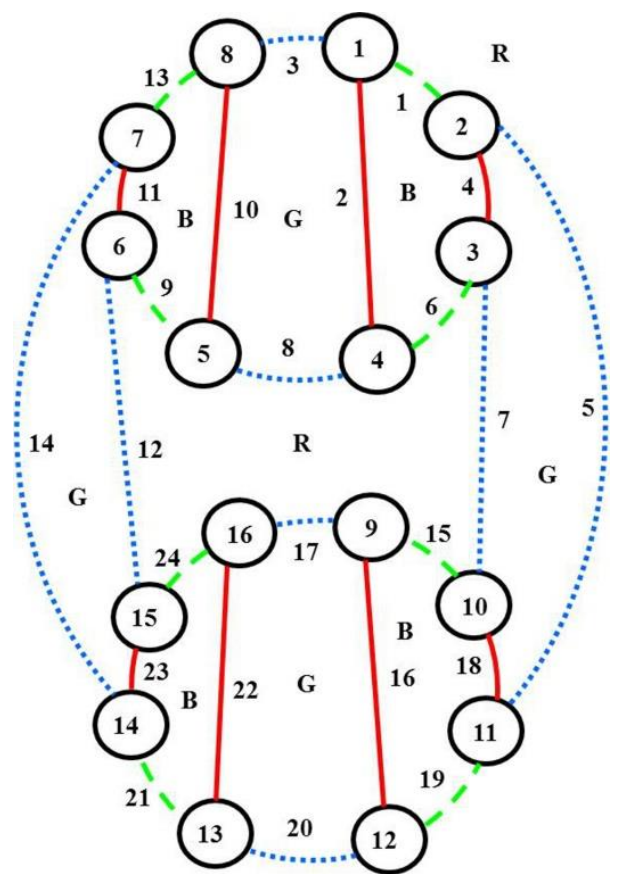

Fig. 12. Recoloring of the cubic graph $\mathbf{H}_{3}$. 
Next, form the rim, $\mathrm{c}_{0}=\left\{\mathrm{e}_{1}[3], \mathrm{e}_{3}[1], \mathrm{e}_{5}[2], \mathrm{e}_{13}[3], \mathrm{e}_{14}[2], \mathrm{e}_{19}[3], \mathrm{e}_{20}[1], \mathrm{e}_{21}[3]\right\}$.

$\mathrm{R}_{\mathrm{c}}=\mathrm{DR}_{1} \oplus \mathrm{DR}_{2}=\left(\mathrm{c}_{3} \oplus \mathrm{c}_{5} \oplus \mathrm{c}_{9}\right) \oplus\left(\mathrm{c}_{2} \oplus \mathrm{c}_{4} \oplus \mathrm{c}_{8}\right)=\left(\left\{\mathrm{e}_{9}[3], \mathrm{e}_{10}[2], \mathrm{e}_{11}[1], \mathrm{e}_{13}[3]\right\} \oplus\right.$

$\left.\oplus\left\{\mathrm{e}_{11}[1], \mathrm{e}_{12}[2], \mathrm{e}_{14}[2], \mathrm{e}_{23}[1]\right\} \oplus\left\{\mathrm{e}_{21}[3], \mathrm{e}_{22}[2], \mathrm{e}_{23}[1], \mathrm{e}_{24}[3]\right\}\right) \oplus\left(\left\{\mathrm{e}_{1}[3], \mathrm{e}_{2}[2], \mathrm{e}_{4}[1], \mathrm{e}_{6}[3]\right\} \oplus\right.$

$\left.\oplus\left\{\mathrm{e}_{4}[1], \mathrm{e}_{5}[2], \mathrm{e}_{7}[2], \mathrm{e}_{18}[1]\right\} \oplus\left\{\mathrm{e}_{15}[3], \mathrm{e}_{16}[2], \mathrm{e}_{18}[1], \mathrm{e}_{19}[3]\right\}\right)=$

$=<\mathrm{e}_{9}[3], \mathrm{e}_{10}[2], \mathrm{e}_{13}[3], \mathrm{e}_{14}[2], \mathrm{e}_{21}[3], \mathrm{e}_{22}[2], \mathrm{e}_{24}[3], \mathrm{e}_{12}[2]>\oplus$

$\oplus<\mathrm{e}_{1}[3], \mathrm{e}_{2}[2], \mathrm{e}_{6}[3], \mathrm{e}_{7}[2], \mathrm{e}_{15}[3], \mathrm{e}_{16}[2], \mathrm{e}_{19}[3], \mathrm{e}_{5}[2]>;$

$\mathrm{B}_{\mathrm{c}}=\mathrm{DB}_{1} \oplus \mathrm{DB}_{2}=\left(\mathrm{c}_{1} \oplus \mathrm{c}_{2} \oplus \mathrm{c}_{3}\right) \oplus\left(\mathrm{c}_{7} \oplus \mathrm{c}_{8} \oplus \mathrm{c}_{9}\right)=\left(\left\{\mathrm{e}_{2}[2], \mathrm{e}_{3}[1], \mathrm{e}_{8}[1], \mathrm{e}_{10}[2]\right\} \oplus\right.$

$\left.\oplus\left\{\mathrm{e}_{1}[3], \mathrm{e}_{2}[2], \mathrm{e}_{4}[1], \mathrm{e}_{6}[3]\right\} \oplus\left\{\mathrm{e}_{9}[3], \mathrm{e}_{10}[2], \mathrm{e}_{11}[1], \mathrm{e}_{13}[3]\right\}\right) \oplus\left(\left\{\mathrm{e}_{16}[2], \mathrm{e}_{17}[1], \mathrm{e}_{20}[1], \mathrm{e}_{22}[2]\right\} \oplus\right.$

$\left.\oplus\left\{\mathrm{e}_{15}[3], \mathrm{e}_{16}[2], \mathrm{e}_{18}[1], \mathrm{e}_{19}[3]\right\} \oplus\left\{\mathrm{e}_{21}[3], \mathrm{e}_{22}[2], \mathrm{e}_{23}[1], \mathrm{e}_{24}[3]\right\}\right)=$

$=<\mathrm{e}_{3}[1], \mathrm{e}_{1}[3], \mathrm{e}_{4}[1], \mathrm{e}_{6}[3], \mathrm{e}_{8}[1], \mathrm{e}_{9}[3], \mathrm{e}_{11}[1], \mathrm{e}_{13}[3]>\oplus$

$\oplus<\mathrm{e}_{17}[1], \mathrm{e}_{15}[3], \mathrm{e}_{18}[1], \mathrm{e}_{19}[3], \mathrm{e}_{20}[1], \mathrm{e}_{21}[3], \mathrm{e}_{23}[1], \mathrm{e}_{24}[3]>$;

$\mathrm{G}_{\mathrm{c}}=\mathrm{DG}_{1} \oplus \mathrm{DG}_{2} \oplus \mathrm{DG}_{3} \oplus \mathrm{DG}_{4}=\left(\mathrm{c}_{1}\right) \oplus\left(\mathrm{c}_{4}\right) \oplus\left(\mathrm{c}_{5}\right) \oplus\left(\mathrm{c}_{7}\right)=<\mathrm{e}_{2}[2], \mathrm{e}_{3}[1], \mathrm{e}_{8}[1], \mathrm{e}_{10}[2]>\oplus$

$\oplus<\mathrm{e}_{4}[1], \mathrm{e}_{5}[2], \mathrm{e}_{7}[2], \mathrm{e}_{18}[1]>\oplus<\mathrm{e}_{11}[1], \mathrm{e}_{12}[2], \mathrm{e}_{14}[2], \mathrm{e}_{23}[1]>\oplus<\mathrm{e}_{16}[2], \mathrm{e}_{17}[1], \mathrm{e}_{20}[1], \mathrm{e}_{22}[2]>$.

$\mathrm{W}_{\mathrm{c}}=\mathrm{c}_{6} \oplus \mathrm{c}_{\mathrm{o}}=\left\{\mathrm{e}_{6}[3], \mathrm{e}_{7}[2], \mathrm{e}_{8}[1], \mathrm{e}_{9}[3], \mathrm{e}_{12}[2], \mathrm{e}_{15}[3], \mathrm{e}_{17}[1], \mathrm{e}_{24}[3]\right\} \oplus$

$\oplus\left\{\mathrm{e}_{1}[3], \mathrm{e}_{3}[1], \mathrm{e}_{5}[2], \mathrm{e}_{13}[3], \mathrm{e}_{14}[2], \mathrm{e}_{19}[3], \mathrm{e}_{20}[1], \mathrm{e}_{21}[3]\right\}$.

Further we carry out the recoloring of edges, taking colored disks instead of white ones as a basis (Fig. 12).

$$
\begin{array}{lrl}
\mathrm{c}_{1}=\left\{\mathrm{e}_{2}[1], \mathrm{e}_{3}[2], \mathrm{e}_{8}[2], \mathrm{e}_{10}[1]\right\}, & \mathrm{c}_{2}=\left\{\mathrm{e}_{1}[3], \mathrm{e}_{2}[1], \mathrm{e}_{4}[1], \mathrm{e}_{6}[3]\right\}, \\
\mathrm{c}_{3}=\left\{\mathrm{e}_{9}[3], \mathrm{e}_{10}[1], \mathrm{e}_{11}[1], \mathrm{e}_{13}[3]\right\}, & \mathrm{c}_{5}=\left\{\mathrm{e}_{11}[1], \mathrm{e}_{12}[2], \mathrm{e}_{14}[2], \mathrm{e}_{23}[1]\right\}, \\
\mathrm{c}_{4}=\left\{\mathrm{e}_{4}[1], \mathrm{e}_{5}[2], \mathrm{e}_{7}[2], \mathrm{e}_{18}[1]\right\}, & \mathrm{c}_{6}=\left\{\mathrm{e}_{6}[3], \mathrm{e}_{7}[2], \mathrm{e}_{8}[2], \mathrm{e}_{9}[3], \mathrm{e}_{12}[2], \mathrm{e}_{15}[3], \mathrm{e}_{17}[2], \mathrm{e}_{24}[3]\right\}, \\
\mathrm{c}_{7}=\left\{\mathrm{e}_{16}[1], \mathrm{e}_{1}[2], \mathrm{e}_{20}[2], \mathrm{e}_{22}[1]\right\}, & \\
\mathrm{c}_{8}=\left\{\mathrm{e}_{15}[3], \mathrm{e}_{16}[1], \mathrm{e}_{18}[1], \mathrm{e}_{19}[3]\right\}, & \mathrm{c}_{9}=\left\{\mathrm{e}_{2_{1}}[3], \mathrm{e}_{22}[1], \mathrm{e}_{23}[1], \mathrm{e}_{24}[3]\right\} .
\end{array}
$$

And determine the rim as $\mathrm{c}_{0}=\left\{\mathrm{e}_{1}[3], \mathrm{e}_{3}[2], \mathrm{e}_{5}[2], \mathrm{e}_{13}[3], \mathrm{e}_{14}[2], \mathrm{e}_{19}[3], \mathrm{e}_{20}[2], \mathrm{e}_{21}[3]\right\}$. $\mathrm{R}_{\mathrm{c}}=\mathrm{DR}_{1} \oplus \mathrm{DR}_{2}=\left(\mathrm{c}_{6}\right) \oplus\left(\mathrm{c}_{0}\right)=\left(\mathrm{c}_{6}\right) \oplus\left(\mathrm{c}_{1} \oplus \mathrm{c}_{2} \oplus \mathrm{c}_{3} \oplus \mathrm{c}_{4} \oplus \mathrm{c}_{5} \oplus \mathrm{c}_{6} \oplus \mathrm{c}_{7} \oplus \mathrm{c}_{8} \oplus \mathrm{c}_{9}\right)=$ $=<\mathrm{e}_{6}[3], \mathrm{e}_{7}[2], \mathrm{e}_{15}[3], \mathrm{e}_{17}[2], \mathrm{e}_{24}[3], \mathrm{e}_{12}[2], \mathrm{e}_{9}[3], \mathrm{e}_{8}[2]>\oplus$

$\oplus<\mathrm{e}_{1}[3], \mathrm{e}_{3}[2], \mathrm{e}_{13}[3], \mathrm{e}_{14}[2], \mathrm{e}_{21}[3], \mathrm{e}_{20}[2], \mathrm{e}_{19}[3], \mathrm{e}_{5}[2]>$;

$\mathrm{B}_{\mathrm{c}}=\mathrm{DB}_{1} \oplus \mathrm{DB}_{2} \oplus \mathrm{DB}_{3} \oplus \mathrm{DB}_{4}=\left(\mathrm{c}_{2}\right) \oplus\left(\mathrm{c}_{3}\right) \oplus\left(\mathrm{c}_{8}\right) \oplus\left(\mathrm{c}_{9}\right)=\left\langle\mathrm{e}_{1}[3], \mathrm{e}_{2}[1], \mathrm{e}_{6}[3], \mathrm{e}_{4}[1]\right\rangle \oplus$ $\oplus<\mathrm{e}_{9}[3], \mathrm{e}_{10}[1], \mathrm{e}_{13}[3], \mathrm{e}_{11}[1]>\oplus<\mathrm{e}_{15}[3], \mathrm{e}_{16}[1], \mathrm{e}_{19}[3], \mathrm{e}_{18}[1]>\oplus<\mathrm{e}_{21}[3], \mathrm{e}_{22}[1], \mathrm{e}_{24}[3], \mathrm{e}_{23}[1]>;$ $\mathrm{G}_{\mathrm{\phi}}=\mathrm{DG}_{1} \oplus \mathrm{DG}_{2} \oplus \mathrm{DG}_{3} \oplus \mathrm{DG}_{4}=\left(\mathrm{c}_{1}\right) \oplus\left(\mathrm{c}_{4}\right) \oplus\left(\mathrm{c}_{5}\right) \oplus\left(\mathrm{c}_{7}\right)=\left\langle\mathrm{e}_{2}[1], \mathrm{e}_{3}[2], \mathrm{e}_{10}[1], \mathrm{e}_{8}[2]\right\rangle \oplus$ $\oplus\left\langle\mathrm{e}_{4}[1], \mathrm{e}_{5}[2], \mathrm{e}_{18}[1], \mathrm{e}_{7}[2]\right\rangle \oplus\left\langle\mathrm{e}_{11}[1], \mathrm{e}_{12}[2], \mathrm{e}_{23}[1], \mathrm{e}_{14}[2]\right\rangle \oplus\left\langle\mathrm{e}_{16}[1], \mathrm{e}_{17}[2], \mathrm{e}_{22}[1], \mathrm{e}_{20}[2]>\right.$.

\section{Coloring non-planar cubic graphs}

An important case is the coloring of cycles in non-planar cubic graphs (Fig. 13).

First, we select basis cycles in the graph:

$$
\begin{array}{lll}
\mathrm{c}_{1}=\left\{\mathrm{e}_{1}, \mathrm{e}_{2}, \mathrm{e}_{4}, \mathrm{e}_{6}\right\} ; & \mathrm{c}_{2}=\left\{\mathrm{e}_{9}, \mathrm{e}_{10}, \mathrm{e}_{11}, \mathrm{e}_{12}\right\} ; & \mathrm{c}_{3}=\left\{\mathrm{e}_{2}, \mathrm{e}_{3}, \mathrm{e}_{8}, \mathrm{e}_{11}\right\} ; \\
\mathrm{c}_{4}=\left\{\mathrm{e}_{4}, \mathrm{e}_{5}, \mathrm{e}_{7}, \mathrm{e}_{10}\right\} ; & \mathrm{c}_{5}=\left\{\mathrm{e}_{6}, \mathrm{e}_{7}, \mathrm{e}_{8}, \mathrm{e}_{9}, \mathrm{e}_{10}\right\} ; & \mathrm{c}_{0}=\left\{\mathrm{e}_{1}, \mathrm{e}_{3}, \mathrm{e}_{5}, \mathrm{e}_{10}, \mathrm{e}_{12}\right\} .
\end{array}
$$

Let's represent colored disks for the given edge coloring in the following form:

$\mathrm{DR}_{1}=\left(\mathrm{e}_{1}, \mathrm{G}\right) \oplus\left(\mathrm{e}_{5}, \mathrm{~B}\right) \oplus\left(\mathrm{e}_{9}, \mathrm{G}\right) \oplus\left(\mathrm{e}_{8}, \mathrm{~B}\right) \oplus\left(\mathrm{e}_{6}, \mathrm{G}\right) \oplus\left(\mathrm{e}_{7}, \mathrm{~B}\right) \oplus\left(\mathrm{e}_{12}, \mathrm{G}\right) \oplus\left(\mathrm{e}_{3}, \mathrm{~B}\right)=$ $=\mathrm{c}_{1} \oplus \mathrm{c}_{2} \oplus \mathrm{c}_{3} \oplus \mathrm{c}_{4}$;

$\mathrm{DB}_{1}=\left(\mathrm{e}_{1}, \mathrm{G}\right) \oplus\left(\mathrm{e}_{4}, \mathrm{R}\right) \oplus\left(\mathrm{e}_{6}, \mathrm{G}\right) \oplus\left(\mathrm{e}_{2}, \mathrm{R}\right)=\mathrm{c}_{1}$;

$\mathrm{DB}_{2}=\left(\mathrm{e}_{9}, \mathrm{G}\right) \oplus\left(\mathrm{e}_{10}, \mathrm{R}\right) \oplus\left(\mathrm{e}_{13}, \mathrm{G}\right) \oplus\left(\mathrm{e}_{11}, \mathrm{R}\right)=\mathrm{c}_{2}$;

$\mathrm{DG}_{1}=\left(\mathrm{e}_{2}, \mathrm{R}\right) \oplus\left(\mathrm{e}_{3}, \mathrm{~B}\right) \oplus\left(\mathrm{e}_{11}, \mathrm{R}\right) \oplus\left(\mathrm{e}_{3}, \mathrm{~B}\right)=\mathrm{c}_{3} ;$

$\mathrm{DG}_{2}=\left(\mathrm{e}_{4}, \mathrm{R}\right) \oplus\left(\mathrm{e}_{7}, \mathrm{~B}\right) \oplus\left(\mathrm{e}_{10}, \mathrm{R}\right) \oplus\left(\mathrm{e}_{5}, \mathrm{~B}\right)=\mathrm{c}_{4}$. 


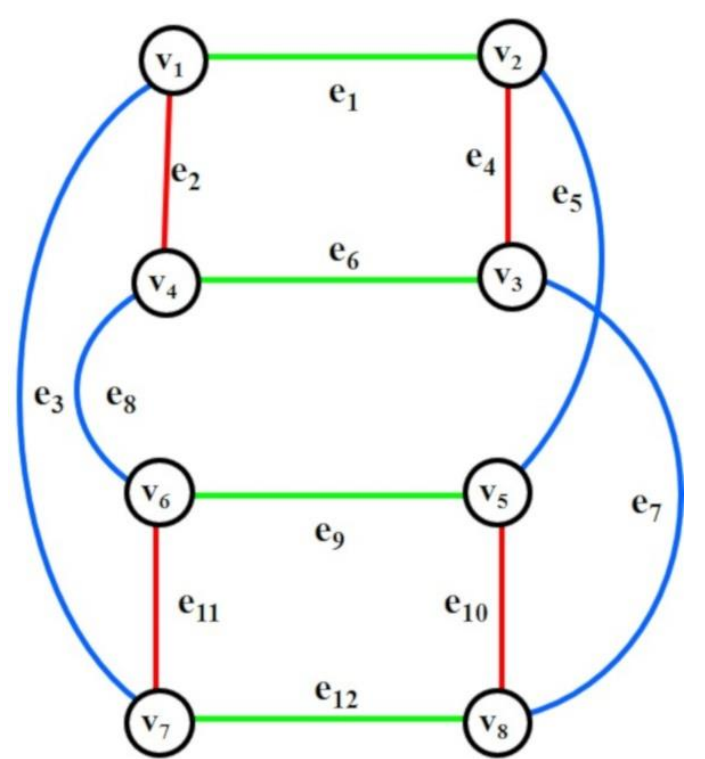

Fig. 13. Colored cubic non-planar graph $\mathbf{H}_{3}$.

The colored 2-factors of the colored cubic non-planar graph $\mathbf{H}_{\mathbf{3}}$ are:

$\mathrm{R}_{\mathrm{c}}=\mathrm{DR}_{1}$;

$\mathrm{B}_{\mathrm{c}}=\mathrm{DB}_{1} \oplus \mathrm{DB}_{2}$;

$\mathrm{G}_{\mathrm{c}}=\mathrm{DG}_{1} \oplus \mathrm{DG}_{2}$;

Thus, we can determine the colors of the basis cycles as follows:
$\mathrm{c}_{1} \rightarrow\left(\mathrm{R}_{\mathrm{c}} \cap \mathrm{B}_{\mathrm{c}}\right) \rightarrow \mathrm{G}$;
$\mathrm{c}_{3} \rightarrow\left(\mathrm{R}_{\mathrm{c}} \cap \mathrm{G}_{\mathrm{c}}\right) \rightarrow \mathrm{B}$;
$\mathrm{c}_{2} \rightarrow\left(\mathrm{R}_{\mathrm{c}} \cap \mathrm{B}_{\mathrm{c}}\right) \rightarrow \mathrm{G}$;
$\mathrm{c}_{5} \rightarrow \mathrm{W}$;
$\mathrm{c}_{4} \rightarrow\left(\mathrm{R}_{\mathrm{c}} \cap \mathrm{G}_{\mathrm{c}}\right) \rightarrow \mathrm{B}$
$\mathrm{c}_{\mathrm{o}} \rightarrow \mathrm{W}$.

Consider the edge coloring:
$\mathrm{e}_{1} \rightarrow\left(\mathrm{c}_{1} \cap \mathrm{c}_{\mathrm{o}}\right) \rightarrow \mathrm{G}+\mathrm{W}=\mathrm{G}$;
$\mathrm{e}_{2} \rightarrow\left(\mathrm{c}_{1} \cap \mathrm{c}_{3}\right) \rightarrow \mathrm{G}+\mathrm{B}=\mathrm{R} ;$
$\mathrm{e}_{3} \rightarrow\left(\mathrm{c}_{3} \cap \mathrm{c}_{\mathrm{o}}\right) \rightarrow \mathrm{B}+\mathrm{W}=\mathrm{B}$;
$\mathrm{e}_{4} \rightarrow\left(\mathrm{c}_{1} \cap \mathrm{c}_{4}\right) \rightarrow \mathrm{G}+\mathrm{B}=\mathrm{R}$;
$\mathrm{e}_{5} \rightarrow\left(\mathrm{c}_{4} \cap \mathrm{c}_{\mathrm{o}}\right) \rightarrow \mathrm{B}+\mathrm{W}=\mathrm{B}$;
$\mathrm{e}_{6} \rightarrow\left(\mathrm{c}_{1} \cap \mathrm{c}_{5}\right) \rightarrow \mathrm{G}+\mathrm{W}=\mathrm{G}$
$\mathrm{e}_{7} \rightarrow\left(\mathrm{c}_{4} \cap \mathrm{c}_{5}\right) \rightarrow \mathrm{B}+\mathrm{W}=\mathrm{B}$;
$\mathrm{e}_{8} \rightarrow\left(\mathrm{c}_{3} \cap \mathrm{c}_{5}\right) \rightarrow \mathrm{B}+\mathrm{W}=\mathrm{B}$;
$\mathrm{e}_{9} \rightarrow\left(\mathrm{c}_{2} \cap \mathrm{c}_{5}\right) \rightarrow \mathrm{G}+\mathrm{W}=\mathrm{G}$;
$\mathrm{e}_{10} \rightarrow\left(\mathrm{c}_{2} \cap \mathrm{c}_{4} \cap \mathrm{c}_{5} \cap \mathrm{c}_{\mathrm{o}}\right) \rightarrow \mathrm{G}+\mathrm{W}+\mathrm{W}+\mathrm{B}=\mathrm{R}$
$\mathrm{e}_{11} \rightarrow\left(\mathrm{c}_{2} \cap \mathrm{c}_{3}\right) \rightarrow \mathrm{G}+\mathrm{B}=\mathrm{R} ; \quad \mathrm{e}_{12} \rightarrow\left(\mathrm{c}_{2} \cap \mathrm{c}_{\mathrm{o}}\right) \rightarrow \mathrm{G}+\mathrm{W}=\mathrm{G}$.

Next, consider the following non-planar graph $\mathbf{H}_{4}$ (Fig. 14). Let's single out the following basis cycles in this graph:
$\mathrm{c}_{1}=\left\{\mathrm{e}_{1}, \mathrm{e}_{2}, \mathrm{e}_{5}, \mathrm{e}_{12}, \mathrm{e}_{15}\right\}$;
$\mathrm{c}_{2}=\left\{\mathrm{e}_{10}, \mathrm{e}_{11}, \mathrm{e}_{12}, \mathrm{e}_{16}\right\} ;$
$\mathrm{c}_{3}=\left\{\mathrm{e}_{1}, \mathrm{e}_{3}, \mathrm{e}_{4}, \mathrm{e}_{7}, \mathrm{e}_{13}\right\} ;$
$\mathrm{c}_{4}=\left\{\mathrm{e}_{4}, \mathrm{e}_{5}, \mathrm{e}_{6}, \mathrm{e}_{9}, \mathrm{e}_{17}\right\} ;$
$\mathrm{c}_{5}=\left\{\mathrm{e}_{6}, \mathrm{e}_{7}, \mathrm{e}_{8}, \mathrm{e}_{11}, \mathrm{e}_{18}\right\} ;$
$\mathrm{c}_{6}=\left\{\mathrm{e}_{8}, \mathrm{e}_{9}, \mathrm{e}_{10}, \mathrm{e}_{12}, \mathrm{e}_{15}, \mathrm{e}_{17}\right\} ;$
$\mathrm{c}_{7}=\left\{\mathrm{e}_{13}, \mathrm{e}_{14}, \mathrm{e}_{15}, \mathrm{e}_{16}, \mathrm{e}_{17}, \mathrm{e}_{18}\right\} ; \mathrm{c}_{\mathrm{o}}=\left\{\mathrm{e}_{2}, \mathrm{e}_{3}, \mathrm{e}_{12}, \mathrm{e}_{14}, \mathrm{e}_{15}, \mathrm{e}_{17}\right\}$. 


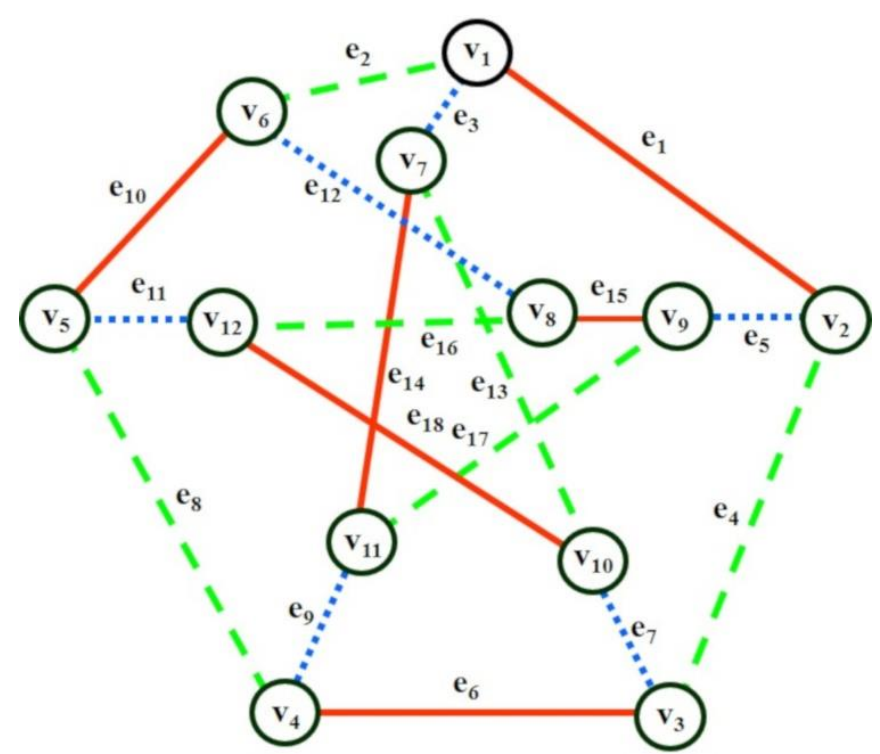

Fig. 14. Colored non-planar graph $\mathbf{H}_{4}$.

The colored disks for this edge coloring are as follows:

$\mathrm{DR}_{1}=\left(\mathrm{e}_{2}, \mathrm{G}\right) \oplus\left(\mathrm{e}_{12}, \mathrm{~B}\right) \oplus\left(\mathrm{e}_{16}, \mathrm{G}\right) \oplus\left(\mathrm{e}_{11}, \mathrm{~B}\right) \oplus\left(\mathrm{e}_{8}, \mathrm{G}\right) \oplus\left(\mathrm{e}_{9}, \mathrm{~B}\right) \oplus\left(\mathrm{e}_{17}, \mathrm{G}\right) \oplus\left(\mathrm{e}_{5}, \mathrm{~B}\right) \oplus$

$\oplus\left(\mathrm{e}_{4}, \mathrm{G}\right) \oplus\left(\mathrm{e}_{7}, \mathrm{~B}\right) \oplus\left(\mathrm{e}_{13}, \mathrm{G}\right) \oplus\left(\mathrm{e}_{3}, \mathrm{~B}\right)=\mathrm{c}_{1} \oplus \mathrm{c}_{2} \oplus \mathrm{c}_{3} \oplus \mathrm{c}_{6} ;$

$\mathrm{DB}_{1}=\left(\mathrm{e}_{1}, \mathrm{R}\right) \oplus\left(\mathrm{e}_{2}, \mathrm{G}\right) \oplus\left(\mathrm{e}_{10}, \mathrm{R}\right) \oplus\left(\mathrm{e}_{8}, \mathrm{G}\right) \oplus\left(\mathrm{e}_{6}, \mathrm{R}\right) \oplus\left(\mathrm{e}_{4}, \mathrm{G}\right)=\mathrm{c}_{1} \oplus \mathrm{c}_{4} \oplus \mathrm{c}_{6}$;

$\mathrm{DB}_{2}=\left(\mathrm{e}_{13}, \mathrm{G}\right) \oplus\left(\mathrm{e}_{18}, \mathrm{R}\right) \oplus\left(\mathrm{e}_{16}, \mathrm{G}\right) \oplus\left(\mathrm{e}_{15}, \mathrm{R}\right) \oplus\left(\mathrm{e}_{17}, \mathrm{G}\right) \oplus\left(\mathrm{e}_{14}, \mathrm{R}\right)=\mathrm{c}_{7}$;

$\mathrm{DG}_{1}=\left(\mathrm{e}_{10}, \mathrm{R}\right) \oplus\left(\mathrm{e}_{11}, \mathrm{~B}\right) \oplus\left(\mathrm{e}_{18}, \mathrm{R}\right) \oplus\left(\mathrm{e}_{7}, \mathrm{~B}\right) \oplus\left(\mathrm{e}_{6}, \mathrm{R}\right) \oplus\left(\mathrm{e}_{9}, \mathrm{~B}\right) \oplus\left(\mathrm{e}_{14}, \mathrm{R}\right) \oplus\left(\mathrm{e}_{3}, \mathrm{~B}\right) \oplus$

$\oplus\left(\mathrm{e}_{1}, \mathrm{R}\right) \oplus\left(\mathrm{e}_{5}, \mathrm{~B}\right) \oplus\left(\mathrm{e}_{15}, \mathrm{R}\right) \oplus\left(\mathrm{e}_{12}, \mathrm{~B}\right)=\mathrm{c}_{2} \oplus \mathrm{c}_{3} \oplus \mathrm{c}_{4} \oplus \mathrm{c}_{7}$.

Colored 2-factors of the colored cubic non-planar graph $\mathbf{H}_{\mathbf{4}}$ are:

$\mathrm{R}_{\mathrm{c}}=\mathrm{DR}_{1}$;

$\mathrm{B}_{\mathrm{c}}=\mathrm{DB}_{1} \oplus \mathrm{DB}_{2}$;

$\mathrm{G}_{\mathrm{c}}=\mathrm{DG}_{1}$;

From this, the colors of the basis cycles can be determined as:
$\mathrm{c}_{1} \rightarrow\left(\mathrm{R}_{\mathrm{c}} \cap \mathrm{B}_{\mathrm{c}}\right) \rightarrow \mathrm{G}$;
$\mathrm{c}_{3} \rightarrow\left(\mathrm{R}_{\mathrm{c}} \cap \mathrm{G}_{\mathrm{c}}\right) \rightarrow \mathrm{B}$
$\mathrm{c}_{2} \rightarrow\left(\mathrm{R}_{\mathrm{c}} \cap \mathrm{G}_{\mathrm{c}}\right) \rightarrow \mathrm{B}$
$\mathrm{c}_{5} \rightarrow \mathrm{W}$
$\mathrm{c}_{4} \rightarrow\left(\mathrm{B}_{\mathrm{c}} \cap \mathrm{G}_{\mathrm{c}}\right) \rightarrow \mathrm{R}$
$\mathrm{c}_{7} \rightarrow\left(\mathrm{B}_{\mathrm{c}} \cap \mathrm{G}_{\mathrm{c}}\right) \rightarrow \mathrm{R}$
$\mathrm{c}_{6} \rightarrow\left(\mathrm{B}_{\mathrm{c}} \cap \mathrm{R}_{\mathrm{c}}\right) \rightarrow \mathrm{G}$
$\mathrm{c}_{\mathrm{o}} \rightarrow \mathrm{W}$.

Consider the edge coloring:

$$
\begin{array}{ll}
\mathrm{e}_{1} \rightarrow\left(\mathrm{c}_{1} \cap \mathrm{c}_{3}\right) \rightarrow \mathrm{G}+\mathrm{B}=\mathrm{R} ; & \mathrm{e}_{2} \rightarrow\left(\mathrm{c}_{1} \cap \mathrm{c}_{0}\right) \rightarrow \mathrm{G}+\mathrm{W}=\mathrm{G} ; \\
\mathrm{e}_{3} \rightarrow\left(\mathrm{c}_{3} \cap \mathrm{c}_{0}\right) \rightarrow \mathrm{B}+\mathrm{W}=\mathrm{B} ; & \mathrm{e}_{4} \rightarrow\left(\mathrm{c}_{3} \cap \mathrm{c}_{4}\right) \rightarrow \mathrm{B}+\mathrm{R}=\mathrm{G} ; \\
\mathrm{e}_{5} \rightarrow\left(\mathrm{c}_{1} \cap \mathrm{c}_{4}\right) \rightarrow \mathrm{G}+\mathrm{R}=\mathrm{B} ; & \mathrm{e}_{6} \rightarrow\left(\mathrm{c}_{4} \cap \mathrm{c}_{5}\right) \rightarrow \mathrm{R}+\mathrm{W}=\mathrm{R} ; \\
\mathrm{e}_{7} \rightarrow\left(\mathrm{c}_{3} \cap \mathrm{c}_{5}\right) \rightarrow \mathrm{B}+\mathrm{W}=\mathrm{B} ; & \mathrm{e}_{8} \rightarrow\left(\mathrm{c}_{5} \cap \mathrm{c}_{6}\right) \rightarrow \mathrm{G}+\mathrm{W}=\mathrm{G} ; \\
\mathrm{e}_{9} \rightarrow\left(\mathrm{c}_{4} \cap \mathrm{c}_{6}\right) \rightarrow \mathrm{R}+\mathrm{G}=\mathrm{B} ; & \mathrm{e}_{10} \rightarrow\left(\mathrm{c}_{2} \cap \mathrm{c}_{6} \rightarrow \mathrm{B}+\mathrm{G}=\mathrm{R} ;\right. \\
\mathrm{e}_{11} \rightarrow\left(\mathrm{c}_{2} \cap \mathrm{c}_{5}\right) \rightarrow \mathrm{B}+\mathrm{W}=\mathrm{B} ; & \\
\mathrm{e}_{12} \rightarrow\left(\mathrm{c}_{1} \cap \mathrm{c}_{2} \cap \mathrm{c}_{6} \cap \mathrm{c}_{0}\right) \rightarrow \mathrm{G}+\mathrm{B}+\mathrm{G}+\mathrm{W}=\mathrm{B} ; & \\
\mathrm{e}_{13} \rightarrow\left(\mathrm{c}_{3} \cap \mathrm{c}_{7}\right) \rightarrow \mathrm{B}+\mathrm{R}=\mathrm{G} ; & \mathrm{e}_{14} \rightarrow\left(\mathrm{c}_{7} \cap \mathrm{c}_{0}\right) \rightarrow \mathrm{R}+\mathrm{W}=\mathrm{R} ; \\
\mathrm{e}_{15} \rightarrow\left(\mathrm{c}_{7} \cap \mathrm{c}_{0}\right) \rightarrow \mathrm{R}+\mathrm{W}=\mathrm{R} ; & \mathrm{e}_{16} \rightarrow\left(\mathrm{c}_{2} \cap \mathrm{c}_{7}\right) \rightarrow \mathrm{B}+\mathrm{R}=\mathrm{G} ; \\
\mathrm{e}_{17} \rightarrow\left(\mathrm{c}_{4} \cap \mathrm{c}_{6} \cap \mathrm{c}_{7} \cap \mathrm{c}_{0}\right) \rightarrow \mathrm{G}+\mathrm{R}+\mathrm{W}+\mathrm{R}=\mathrm{G} ; & \\
\mathrm{e}_{18} \rightarrow\left(\mathrm{c}_{5} \cap \mathrm{c}_{7}\right) \rightarrow \mathrm{R}+\mathrm{W}=\mathrm{R} . &
\end{array}
$$

Hence, Formula 5 is also applicable to colored non-planar graphs. 


\section{Colored rotation of vertices}

A cyclic order of the form $1-2-3(\mathrm{R}-\mathrm{B}-\mathrm{G})$ of the traversal of edges for a vertex of a plane cubic graph $\mathbf{H}$ is further denoted as the colored rotation of vertices. If the edges of a vertex are colored with three colors, then the order of traversing the colors $1-2-3(R-B-G)$ for colored edges can be either clockwise or counterclockwise. We put " +1 " in correspondence with those vertices for which color rotation occurs clockwise, and "-1" - with those for which the rotation direction is opposite. Consider the following color rotation of the vertices shown in Fig. 15.

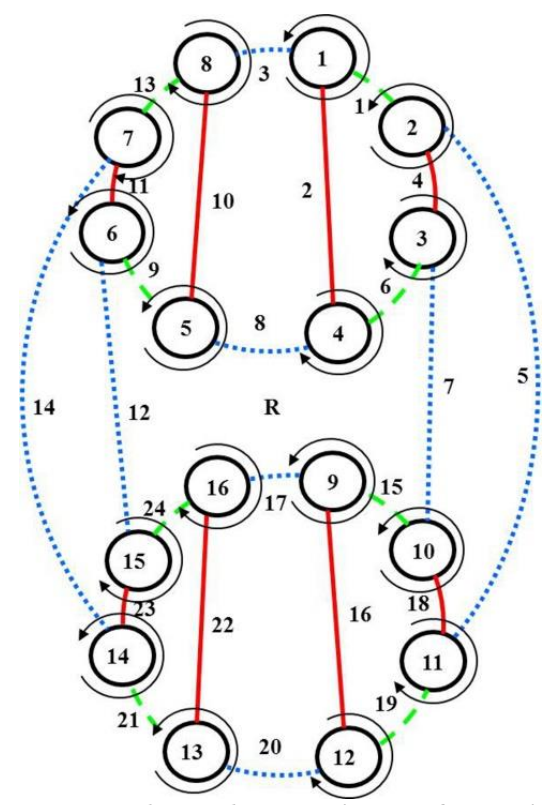

Fig. 15. Colored rotation of vertices.

Then, for the set of vertices belonging to the face of a plane cubic graph, we can write the Heawood system of equations [1] for each basis cycle and rim:

$$
\left\{\begin{array}{l}
x_{i}^{2} \equiv 0(\bmod 3) \\
\sum_{i \in M_{\alpha}} x_{i} \equiv 0(\bmod 3)
\end{array}\right.
$$

where $\alpha=1,2, \ldots, m-n+2$.

At the same time, the solution of the Heawood system determines the coloring, since in the Heawood system the clockwise colored rotation of the vertices of a plane cubic graph can be put in correspondence with “ +1 ”, and the counterclockwise colored rotation - with " -1 ”. Thus, it is possible to write the Heawood equations [1] in the following form:

$$
\begin{aligned}
& \mathrm{x}\left(\mathrm{v}_{1}\right)+\mathrm{x}\left(\mathrm{v}_{4}\right)+\mathrm{x}\left(\mathrm{v}_{5}\right)+\mathrm{x}\left(\mathrm{v}_{8}\right)=(-1)+(+1)+(-1)+(+1) \equiv \mathrm{o}(\bmod 3) \\
& \mathrm{x}\left(\mathrm{v}_{1}\right)+\mathrm{x}\left(\mathrm{v}_{2}\right)+\mathrm{x}\left(\mathrm{v}_{3}\right)+\mathrm{x}\left(\mathrm{v}_{4}\right)=(-1)+(-1)+(+1)+(+1) \equiv \mathrm{o}(\bmod 3) \\
& \mathrm{x}\left(\mathrm{v}_{5}\right)+\mathrm{x}\left(\mathrm{v}_{6}\right)+\mathrm{x}\left(\mathrm{v}_{7}\right)+\mathrm{x}\left(\mathrm{v}_{8}\right)=(-1)+(-1)+(+1)+(+1) \equiv \mathrm{o}(\bmod 3) \\
& \mathrm{x}\left(\mathrm{v}_{2}\right)+\mathrm{x}\left(\mathrm{v}_{3}\right)+\mathrm{x}\left(\mathrm{v}_{10}\right)+\mathrm{x}\left(\mathrm{v}_{11}\right)=(-1)+(+1)+(-1)+(+1) \equiv \mathrm{O}(\bmod 3) \\
& \mathrm{x}\left(\mathrm{v}_{6}\right)+\mathrm{x}\left(\mathrm{v}_{7}\right)+\mathrm{x}\left(\mathrm{v}_{14}\right)+\mathrm{x}\left(\mathrm{v}_{15}\right)=(-1)+(+1)+(-1)+(+1) \equiv \mathrm{O}(\bmod 3) \\
& \mathrm{x}\left(\mathrm{v}_{3}\right)+\mathrm{x}\left(\mathrm{v}_{4}\right)+\mathrm{x}\left(\mathrm{v}_{5}\right)+\mathrm{x}\left(\mathrm{v}_{6}\right)+\mathrm{x}\left(\mathrm{v}_{9}\right)+\mathrm{x}\left(\mathrm{v}_{10}\right)+\mathrm{x}\left(\mathrm{v}_{15}\right)+\mathrm{x}\left(\mathrm{v}_{16}\right)= \\
& =(+1)+(+1)+(-1)+(-1)+(-1)+(-1)+(+1)+(+1) \equiv \mathrm{o}(\bmod 3) \\
& \mathrm{x}\left(\mathrm{v}_{9}\right)+\mathrm{x}\left(\mathrm{v}_{12}\right)+\mathrm{x}\left(\mathrm{v}_{13}\right)+\mathrm{x}\left(\mathrm{v}_{16}\right)=(-1)+(+1)+(-1)+(+1) \equiv \mathrm{o}(\bmod 3) \\
& \mathrm{x}\left(\mathrm{v}_{9}\right)+\mathrm{x}\left(\mathrm{v}_{10}\right)+\mathrm{x}\left(\mathrm{v}_{11}\right)+\mathrm{x}\left(\mathrm{v}_{12}\right)=(-1)+(-1)+(+1)+(+1) \equiv \mathrm{o}(\bmod 3) \\
& \mathrm{x}\left(\mathrm{v}_{13}\right)+\mathrm{x}\left(\mathrm{v}_{14}\right)+\mathrm{x}\left(\mathrm{v}_{15}\right)+\mathrm{x}\left(\mathrm{v}_{16}\right)=(-1)+(-1)+(+1)+(+1) \equiv \mathrm{O}(\bmod 3) \\
& \mathrm{x}\left(\mathrm{v}_{1}\right)+\mathrm{x}\left(\mathrm{v}_{2}\right)+\mathrm{x}\left(\mathrm{v}_{7}\right)+\mathrm{x}\left(\mathrm{v}_{8}\right)+\mathrm{x}\left(\mathrm{v}_{11}\right)+\mathrm{x}\left(\mathrm{v}_{12}\right)+\mathrm{x}\left(\mathrm{v}_{13}\right)+\mathrm{x}\left(\mathrm{v}_{14}\right)=
\end{aligned}
$$


$=(-1)+(-1)+(+1)+(+1)+(+1)+(+1)+(-1)+(-1) \equiv 0(\bmod 3)$

The following theorem holds for maximal planar graphs.

Theorem 1 [4]. Let $\mathrm{M}$ be the set of vertices of a plane cubic graph. Then for any correct coloring

Indeed,

$$
\sum_{\mu \in M} x_{\mu} \equiv 0(\bmod 4)
$$

$\mathrm{x}\left(\mathrm{v}_{1}\right)+\mathrm{x}\left(\mathrm{v}_{2}\right)+\mathrm{x}\left(\mathrm{v}_{3}\right)+\mathrm{x}\left(\mathrm{v}_{4}\right)+\mathrm{x}\left(\mathrm{v}_{5}\right)+\mathrm{x}\left(\mathrm{v}_{6}\right)+\mathrm{x}\left(\mathrm{v}_{7}\right)+\mathrm{x}\left(\mathrm{v}_{8}\right)+\mathrm{x}\left(\mathrm{v}_{9}\right)+\mathrm{x}\left(\mathrm{v}_{10}\right)+\mathrm{x}\left(\mathrm{v}_{11}\right)+\mathrm{x}\left(\mathrm{v}_{12}\right)+$ $\mathrm{x}\left(\mathrm{v}_{13}\right)+\mathrm{x}\left(\mathrm{v}_{14}\right)+\mathrm{x}\left(\mathrm{v}_{15}\right)+\mathrm{x}\left(\mathrm{v}_{16}\right)=(-1)+(-1)+(+1)+(+1)+(-1)+(+1)+(+1)+(-1)+(-1)+(-1)$ $+(+1)+(+1)+(-1)+(-1)+(+1)+(+1) \equiv 0(\bmod 4)$.

Thus, we can construct closed Heawood paths in a plane colored cubic graph, sequentially selecting edges according to the color rotation of the vertices.

$$
\begin{aligned}
& \mu_{1}=<\mathrm{e}_{3}[1], \mathrm{e}_{10}[2], \mathrm{e}_{9}[3], \mathrm{e}_{11}[1], \mathrm{e}_{14}[2], \mathrm{e}_{21}[3], \mathrm{e}_{20}[1], \mathrm{e}_{16}[2], \mathrm{e}_{15}[3], \mathrm{e}_{18}[1], \mathrm{e}_{5}[2], \mathrm{e}_{1}[3]>; \\
& \mu_{2}=<\mathrm{e}_{3}[1], \mathrm{e}_{2}[2], \mathrm{e}_{6}[3], \mathrm{e}_{4}[1], \mathrm{e}_{5}[2], \mathrm{e}_{19}[3], \mathrm{e}_{20}[1], \mathrm{e}_{22}[2], \mathrm{e}_{24}[3], \mathrm{e}_{23}[1], \mathrm{e}_{14}[2], \mathrm{e}_{13}[3]>; \\
& \mu_{3}=<\mathrm{e}_{23}[1], \mathrm{e}_{12}[2], \mathrm{e}_{9}[3], \mathrm{e}_{8}[1], \mathrm{e}_{2}[2], \mathrm{e}_{1}[3], \mathrm{e}_{4}[1], \mathrm{e}_{7}[2], \mathrm{e}_{15}[3], \mathrm{e}_{17}[1], \mathrm{e}_{22}[2], \mathrm{e}_{21}[3]>; \\
& \mu_{4}=<\mathrm{e}_{11}[1], \mathrm{e}_{12}[2], \mathrm{e}_{24}[3], \mathrm{e}_{17}[1], \mathrm{e}_{16}[2], \mathrm{e}_{19}[3], \mathrm{e}_{18}[1], \mathrm{e}_{7}[2], \mathrm{e}_{6}[3], \mathrm{e}_{8}[1], \mathrm{e}_{10}[2], \mathrm{e}_{13}[3]>
\end{aligned}
$$

Heawood closed paths pass through an edge twice, but the second time is always in the opposite direction. The length of the Heawood closed path is a multiple of three.

\section{Conclusion}

The article addresses algebraic methods for coloring arbitrary - both plane and non-planar cubic graphs. The methods are partially based on the corollaries of the Tait's theorem and the coloring of a plane cubic graph is described using the fourth-order Klein group transformation. It is established that the topological drawing of a plane graph, which is formally described by vertices rotation, induces basis simple cycles of the cycle subspace of a graph. The transition to graph coloring is done by coloring the edges of basis cycles. It is assumed that the coloring was made by the methods presented in [3]. Details on the formation of colored disks based on the coloring of edges are presented in the article. The authors introduce the concept of embeddability of colored disks in order to unambiguously describe the representation of colored disks by means of basis cycles. In addition, the issues related to the mathematical description of colored disks rotation operation and their subsequent recoloring are considered in the article. The relationship between the colored vertex rotation of a plane cubic graph and the closed Heawood paths is revealed and formally described.

\section{References}

1. A.A. Zykov. Fundamentals of Graph Theory. - B C S Associates, 1990. -365 p.

2. I. Grossman, W Magnus. In Groups and Their Graphs. - Mathematical Association of America, 1992. - 195 p.

3. Kurapov $S$. V, Davidovsky $M$. V. Visual algorithm for coloring planar graphs // Scientific Visualization. - 2018, Vol. 10, No 3- P. 1-33.

4. N. Z. Shor, G. A. Donets. Algebraic Approach to the Plane Graph Coloring. - Naukova Dumka, 1982. English translation published by Springer-Verlag, Berlin, 1985.

5. G. Ringel. Map Color Theorem. - Repr. of the orig. 1st ed. (1974), Springer, 2011. - 212 p.

6. M. N. S. Swamy. Graphs, networks, and algorithms / Swamy, M.N.S., Thulasiraman K. Wiley New York, 1981. - 592 p.

7. P. G. Tait. Remarks on the Colourings of Maps // In Proc. R. Soc. Edinburgh 10: 729, 1880. 